\title{
Heterocomplexes between the Atypical Chemokine MIF and the CXC-Motif Chemokine CXCL4L1 Regulate Inflammation and Thrombus Formation
}

\section{Markus Brandhofer}

Ludwig-Maximilians-Universität München: Ludwig-Maximilians-Universitat Munchen

\section{Adrian Hoffmann}

Ludwig-Maximilians-Universität München: Ludwig-Maximilians-Universitat Munchen

\section{Xavier Blanchet}

Ludwig-Maximilians-Universität München: Ludwig-Maximilians-Universitat Munchen

\section{Elena Siminkovitch}

Ludwig-Maximilians-Universität München: Ludwig-Maximilians-Universitat Munchen

\section{Anne-Katrin Rohlfing}

Universitätsklinikum Tübingen: Universitatsklinikum Tubingen

\section{Omar El Bounkari}

Ludwig-Maximilians-Universität München: Ludwig-Maximilians-Universitat Munchen

Jeremy A. Nestele

Universitätsklinikum Tübingen: Universitatsklinikum Tubingen

\section{Alexander Bild}

Universitätsklinikum Tübingen: Universitatsklinikum Tubingen

\section{Christos Kontos}

Technische Universität München: Technische Universitat Munchen

\section{Kathleen Hille}

Technische Universität München: Technische Universitat Munchen

\section{Vanessa Rohde}

Ludwig-Maximilians-Universität München: Ludwig-Maximilians-Universitat Munchen

\section{Adrian Fröhlich}

Ludwig-Maximilians-Universität München: Ludwig-Maximilians-Universitat Munchen Jona Golemi

Ludwig-Maximilians-Universität München: Ludwig-Maximilians-Universitat Munchen

\section{Ozgun Gokce}

Ludwig-Maximilians-Universität München: Ludwig-Maximilians-Universitat Munchen

\section{Christine Krammer}

Ludwig-Maximilians-Universität München: Ludwig-Maximilians-Universitat Munchen 
Ludwig-Maximilians-Universität München: Ludwig-Maximilians-Universitat Munchen

\section{Nikolaos Tsilimparis}

Ludwig-Maximilians-Universität München: Ludwig-Maximilians-Universitat Munchen

\section{Wolfgang E. Kempf}

Technische Universität München: Technische Universitat Munchen

\section{Lars Maegdefessel}

Technische Universität München: Technische Universitat Munchen

\section{Remco T.A. Megens}

Ludwig-Maximilians-Universität München: Ludwig-Maximilians-Universitat Munchen

\section{Hans Ippel}

Maastricht University Cardiovascular Research Institute Maastricht: Universiteit Maastricht Cardiovascular Research Institute Maastricht

\section{Rory R. Koenen}

Maastricht University Cardiovascular Research Institute Maastricht: Universiteit Maastricht

Cardiovascular Research Institute Maastricht

\section{Kevin H. Mayo}

University of Minnesota Medical Center: University of Minnesota Health

\section{Meinrad Gawaz}

Universitätsklinikum Tübingen: Universitatsklinikum Tubingen

\section{Aphrodite Kapurniotu}

Technische Universität München: Technische Universitat Munchen

\section{Christian Weber}

Ludwig-Maximilians-Universität München: Ludwig-Maximilians-Universitat Munchen

\section{Philipp von Hundelshausen}

Ludwig-Maximilians-Universität München: Ludwig-Maximilians-Universitat Munchen

\section{Jürgen Bernhagen ( $\sim$ juergen.bernhagen@med.uni-muenchen.de)}

Ludwig-Maximilians-Universität München: Ludwig-Maximilians-Universitat Munchen https://orcid.org/0000-0003-2996-2652

\section{Research Article}

\section{Keywords:}

Posted Date: February 7th, 2022

DOI: https://doi.org/10.21203/rs.3.rs-1300172/v1

License: (c) (i) This work is licensed under a Creative Commons Attribution 4.0 International License. Read Full License 


\section{Heterocomplexes between the Atypical Chemokine MIF and the} CXC-Motif Chemokine CXCL4L1 Regulate Inflammation and Thrombus Formation

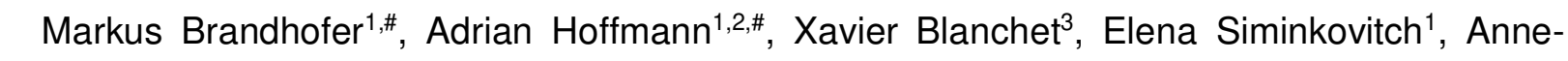
Katrin Rohlfing ${ }^{4}$, Omar El Bounkari ${ }^{1}$, Jeremy A. Nestele ${ }^{4}$, Alexander Bild ${ }^{4}$, Christos Kontos ${ }^{5}$, Kathleen Hille ${ }^{5}$, Vanessa Rohde ${ }^{1}$, Adrian Fröhlich ${ }^{1}$, Jona Golemi ${ }^{6}$, Ozgun Gokce ${ }^{6,7}$, Christine Krammer $^{1}$, Patrick Scheiermann², Nikolaos Tsilimparis ${ }^{8}$, Wolfgang E. Kempf ${ }^{9}$, Lars Maegdefessel $^{9}$, Remco T.A. Megens ${ }^{3}$, Hans Ippel ${ }^{10}$, Rory R. Koenen ${ }^{10}$, Kevin H. Mayo ${ }^{10,11}$, Meinrad Gawaz ${ }^{4}$, Aphrodite Kapurniotu ${ }^{5}$, Christian Weber ${ }^{3,7,10,12}$, Philipp von Hundelshausen $^{3, *}$, Jürgen Bernhagen ${ }^{1,7,12 *}$

${ }^{1}$ Division of Vascular Biology, Institute for Stroke and Dementia Research (ISD), LMU University Hospital, Ludwig-Maximilians-Universität (LMU) München, 81377 Munich, Germany; ${ }^{2}$ Department of Anesthesiology, LMU University Hospital, Ludwig-MaximiliansUniversität (LMU) München, 81377 Munich, Germany; ${ }^{3}$ Institute for Cardiovascular Prevention, LMU University Hospital, Ludwig-Maximilians-Universität (LMU) München, 80336 Munich, Germany; ${ }^{4}$ Department of Cardiology and Angiology, University Hospital Tübingen, Eberhard Karls University Tübingen, Tübingen, Germany; ${ }^{5}$ Division of Peptide Biochemistry, TUM School of Life Sciences, Technische Universität München (TUM); ${ }^{6}$ Systems Neuroscience Group, Institute for Stroke and Dementia Research (ISD), LMU University Hospital, Ludwig-Maximilians-Universität (LMU) München, 81377 Munich, Germany; ${ }^{7}$ Munich Cluster for Systems Neurology (SyNergy), 81377 Munich, Germany; ${ }^{8}$ Department of Vascular Surgery, LMU University Hospital, Ludwig-Maximilians-Universität (LMU) München, 81377 Munich, Germany; ${ }^{9}$ Department for Vascular and Endovascular Surgery, Klinikum rechts der Isar, Technische Universität München (TUM), 81675 Munich, Germany; ${ }^{10}$ Cardiovascular Research Institute Maastricht (CARIM), Maastricht University, 6229 ER Maastricht, The Netherlands; ${ }^{11}$ Department of Biochemistry, Molecular Biology and Biophysics, Health 
28 Sciences Center, University of Minnesota, Minneapolis, MN, USA; ${ }^{12}$ Munich Heart Alliance, 2980802 Munich, Germany.

Correspondence:

Jürgen Bernhagen, PhD

Professor and Chair of Vascular Biology

Institute for Stroke and Dementia Research (ISD)

LMU University Hospital (LMU Klinikum)

Ludwig-Maximilians-Universität (LMU) München

Feodor-Lynen-Straße 17, 81377 Munich, Germany

Tel.: $\quad 0049-894400-46151$

E-Mail: juergen.bernhagen@med.uni-muenchen.de

Philipp von Hundelshausen, MD

Institute for Cardiovascular Prevention (IPEK)

LMU University Hospital (LMU Klinikum)

Ludwig-Maximilians-Universität (LMU) München

Pettenkofer Straße 8a/9, 80336 Munich, Germany

Tel.: $\quad 0049-894400-54359$

E-Mail: Philipp.von Hundelshausen@med.uni-muenchen.de 


\section{Abstract}

34 To fulfil its orchestrating function in immune cell trafficking, a network of chemokines and receptors capitalizes on specificity, redundancy, and functional selectivity. The discovery of heteromeric interactions in the chemokine interactome has expanded the complexity within the network. Moreover, some inflammatory mediators, not structurally linked to classical chemokines, bind to chemokine receptors and behave as atypical chemokines (ACKs). We identified macrophage migration inhibitory factor (MIF) as an ACK that binds to the chemokine receptors CXCR2 and CXCR4 to promote atherogenic leukocyte recruitment. Here, we hypothesized that chemokine-chemokine interactions extend to ACKs and that MIF may form heterocomplexes with classical chemokines. We tested this hypothesis by an unbiased chemokine protein array. The platelet chemokine CXCL4L1, but not its variant CXCL4 or the CXCR2/CXCR4 ligands CXCL8 or CXCL12, was identified as a candidate interactor. MIF/CXCL4L1 complexation was verified by co-immunoprecipitation, surface plasmonresonance analysis, and microscale thermophoresis, also establishing high-affinity binding. We next determined whether heterocomplex formation modulates inflammatory/atherogenic activities of MIF. Complex formation abrogated MIF-elicited T-cell chemotaxis as assessed in a 3D-matrix-based live cell-imaging set-up. Heterocomplexation also blocked MIF-triggered migration of microglia in cortical cultures in situ. Of note, CXCL4L1 blocked the binding of Alexa-MIF to a soluble surrogate of CXCR4 and co-incubation with CXCL4L1 attenuated MIF responses in HEK293-CXCR4 transfectants, indicating that complex formation interferes with MIF/CXCR4 pathways. As MIF and CXCL4L1 are platelet products, we finally tested their role in platelet activation. Multi-photon microscopy, FLIM-FRET, and proximity-ligation assay visualized heterocomplexes in platelet aggregates and clinical human thrombus sections. Moreover, heterocomplexes inhibited MIF-stimulated thrombus formation under flow and skewed the lamellipodia phenotype of adhering platelets. Our study establishes a novel molecular interaction, adding to the complexity of the chemokine interactome and chemokine/receptor-network. MIF/CXCL4L1, or more generally, ACK/CXC-motif chemokine heterocomplexes may be target structures to modulate inflammation and thrombosis. 


\section{Introduction}

Chemokines orchestrate immune cell trafficking in health and disease (Charo \& Ransohoff, 2006; Noels et al, 2019; Weber \& Noels, 2011). Chemokine-directed targeting strategies are pursued in acute and chronic inflammatory conditions, autoimmunity, cancer, and atherosclerosis (Hutchings et al, 2017; Noels et al., 2019; Zlotnik et al, 2011). The chemokine network encompasses 49 classical chemokines (CKs) and 18 classical chemokine receptors (CKRs), which belong to the class of Gia protein-coupled receptors (GPCRs) (Bachelerie et al, 2014a; Bachelerie et al, 2014b; Murphy et al, 2000). Depending on the particular chemokine ligand/receptor pair and various disease and microenvironmental factors, chemokine signaling through CKRs overall capitalizes on the principles of specificity, promiscuity, and biased agonism. Accordingly, multiple chemokines can bind to a certain chemokine receptor and vice versa, while 'biased agonism' can occur on a ligand, receptor, or tissue basis (Eiger et al, 2021; Kleist et al, 2016; Steen et al, 2014). Fine-tuning of chemokine responses within this network is further expanded by five atypical chemokine receptors (ACKRs) that serve as decoy receptors and promiscuously bind many chemokines to shape their gradients, but also elicit specific signaling responses (Nibbs \& Graham, 2013). Chemokines are well-known to form homodimers, but the discovery of the chemokine interactome additionally suggested a multitude of heteromeric chemokine-chemokine interactions even across CC- and CXC-chemokine class borders (Koenen et al, 2009; von Hundelshausen et al, 2017). CC-type heterodimers between CCL5 and CCL17 or CCL5 and CXCL4 (also termed platelet factor 4, PF4) were found to lead to functional synergism by receptor retention or auxiliary proteoglycan binding and enhancement of chemotactic responses, respectively, while CXC-type heterodimers between CXCL12 and CCL5 or CXCL12 and CXCL4 led to signaling inhibition. This has demonstrated yet another level of complexity within the chemokine network and offers novel intervention strategies in inflammatory and cardiovascular diseases (von Hundelshausen et al., 2017).

Moreover, some alarmin-like inflammatory mediators such as human $\beta$-defensins (HBDs) and secreted fragments of amino acyl tRNA-synthetases (AARSs), which do not 
belong to one of the four structural classes of CC-, $\mathrm{CXC}-, \mathrm{CX}_{3} \mathrm{C}$-, or $\mathrm{C}$-chemokines, can bind to chemokine receptors by molecular mimicry and exhibit chemokine-like activities (Rohrl et al, 2010; Wakasugi \& Schimmel, 1999). These proteins are also referred to as atypical chemokines (ACKs) (Degryse \& de Virgilio, 2003; Kapurniotu et al, 2019; Oppenheim \& Yang, 2005).

Macrophage migration inhibitory factor (MIF) is an evolutionarily conserved pleiotropic inflammatory cytokine (David, 1966; Michelet et al, 2019). MIF is an upstream regulator of the host innate immune response and, when dysregulated, is a pivotal mediator of inflammatory diseases, autoimmunity, cancer, and cardiovascular diseases (Calandra \& Roger, 2003; Tilstam et al, 2017). MIF is a structurally unique cytokine (Sun et al, 1996) and, contrary to its eponymous name, has chemokine-like activities and functions as a prototypical ACK (Bernhagen et al, 2007; Kapurniotu et al., 2019). Accordingly, MIF not only signals through its cognate receptor CD74/invariant chain, but engages in high-affinity interactions with the CXC chemokine receptors CXCR2 and CXCR4 to promote atherogenic monocyte and T-/B-cell recruitment, cancer metastasis, and inflammation (Bernhagen et al., 2007; Kapurniotu et al., 2019; Klasen et al, 2014; Leng et al, 2003; Pawig et al, 2015; Sinitski et al, 2019; Tillmann et al, 2013). We elucidated the structural determinants of the binding interface between MIF and its CXC-motif chemokine receptors and found that MIF mimics chemokine receptor binding regions such as the ELR motif and the $\mathrm{N}$-loop (Kraemer et al, 2011b; Krammer et al, 2021; Lacy et al, 2018; Rajasekaran et al, 2016; Weber et al, 2008). Interestingly, CXCL12/SDF-1 $\alpha$ (stromal-derived factor-1 $\alpha$ ), the cognate ligand of CXCR4, was recently found to bind to the non-chemokine proteins galectin-3 (Eckardt et al, 2020) and high-mobility group box-1 (HMGB1) (De Leo et al, 2019; Schiraldi et al, 2012), but potential interactions between MIF and CXCL12 or CXCL8, the cognate ligand of its chemokine receptors CXCR2, have remained unclear.

Here, we hypothesized that chemokine-chemokine interactions are not only possible between different types of classical chemokines, as demonstrated by chemokine interactome mapping (von Hundelshausen et al., 2017), but might extend to ACKs. Choosing MIF as a 
118 prototypical ACK, we thus asked whether this mediator would form heterocomplexes with 119 classical chemokines. We tested this hypothesis applying an unbiased chemokine protein 120 array and validated candidate interactors by a battery of biochemical and biophysical 121 methods. We identified the platelet chemokine CXCL4L1 (also termed PF4var1), but not its 122 variant CXCL4, nor the CXCR2 ligand CXCL8 or the CXCR4 ligand CXCL12, as a high 123 affinity interactor of MIF and tested the potential functional role of CXCL4L1/MIF 124 heterocomplex formation for MIF binding to its receptor CXCR4, and in cell systems that are 125 relevant for the inflammatory, atherogenic, and thrombogenic activities of MIF. Finally, we 126 also asked whether such heterocomplexes can be detected in clinical thrombus specimens. 127 Our study extends the chemokine interactome to ACK/CK interactions and demonstrates a 128 functional role for the MIF/CXCL4L1 heterocomplex in disease-relevant activities. 


\section{Materials and Methods}

132

133

134

\section{Proteins and reagents}

Biologically active and endotoxin-free recombinant human MIF was prepared as previously described and was obtained at a purity of $\sim 98 \%$ as confirmed by SDS-PAGE analysis in combination with silver staining (Bernhagen et al, 1994; Kontos et al, 2020). For the preparation of Alexa Fluor-488- and MST-Red-labeled MIF, a 90-95\% pure MIF fraction was used. Alexa Fluor-488-labeled MIF was generated using the Microscale Protein Labeling Kit from Invitrogen-Molecular Probes (Karlsruhe, Germany) and MST-Red-MIF was prepared using the Monolith Protein Labeling Kit RED-NHS $2^{\text {nd }}$ Generation from NanoTemper (Munich, Germany), following the manufacturers' instructions. Biotinylated human MIF was produced

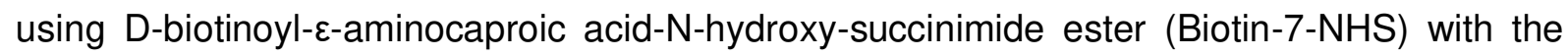

Biotin Protein Labeling Kit from Roche (Mannheim, Germany). Alternatively, biotinamidohexanoic acid N-hydroxysuccinimide ester from Sigma-Aldrich (Taufkirchen, Germany) was used.

For the fluorescence polarization assay, a hexahistidine-tagged variant of CXCL4L1 was used. Briefly, the coding sequence of human CXCL4L1 with a methionine-flanked Nterminal $\mathrm{His}_{6}$-tag was cloned into the $\mathrm{pET} 21$ a vector for recombinant bacterial expression using Xhol and Ndel restriction sites. This construct was then used to transform Rosettagami $^{\mathrm{TM}} 2$ (DE3) competent E. coli (Novagen, Merck KGaA, Darmstadt, Germany) for subsequent recombinant protein production following induction with $1 \mathrm{mM}$ IPTG (Carl Roth, Karlsruhe, Germany) according to a standard protocol. For purification, bacteria pellets were resuspended in lysis buffer $(20 \mathrm{mM}$ Tris- $\mathrm{HCl}, \mathrm{pH} 8.0,0.5 \mathrm{M} \mathrm{NaCl}, 5 \mathrm{mM}$ EDTA, $0.1 \%$ Triton X-100, with added protease inhibitor tablets according to manufacturer's instructions) and cells disrupted in a EmlsiFlex-C5 high pressure homogenizer (Avestin Europe GmbH, Mannheim, Germany), the raw extract cleared via centrifugation at $20.000 \times g$ and the resulting pellet, containing recombinant His-CXCL4L1 in inclusion bodies, was washed in lysis buffer with and without detergent. Inclusion bodies were solubilized by gentle shaking 
159

160

overnight in $50 \mathrm{mM}$ Tris- $\mathrm{HCl}, \mathrm{pH} 8.0,6 \mathrm{M}$ guanidine- $\mathrm{HCl}, 0.5 \mathrm{M} \mathrm{NaCl}, 10 \mathrm{mM}$ DTT and HisCXCL4L1 purified from the solubilized pellet via IMAC on a HisTrap HP column on an ÄKTA Pure 25 M FPLC system (Cytiva Europe GmbH, Freiburg, Germany). The obtained protein was subjected to two dialysis steps in refolding buffer $(50 \mathrm{mM}$ Tris- $\mathrm{HCl}, \mathrm{pH} 8.0,0.5 \mathrm{M} \mathrm{NaCl}$, $5 \mathrm{mM}$ methionine, $5 \mathrm{mM}$ cysteine) with and subsequently without $0.9 \mathrm{M}$ guanidine- $\mathrm{HCl}$, followed by a final purification step by size exclusion chromatography in $20 \mathrm{mM}$ sodium phosphate buffer, pH 7.4, using a Superdex 75 10/300 GL column (Cytiva Europe GmbH) on an ÄKTA Pure 25 M FPLC system. A purity degree of $90-95 \%$ was verified by SDS-PAGE followed by Coomassie staining and Western Blot according to standard protocols.

Recombinant human peroxiredoxins 1 and 6 (PRX1, PRX6) were purchased from Abcam (Abcam PLC, Cambridge, UK), while recombinant human $\beta$-defensin-1 and 2 (HBD-1, HBD-2) were obtained from ProSpec (ProSpec-Tany TechnoGene Ltd., Ness Ziona, Israel). Recombinant human HMGB1 was purchased from Novus (Novus Biologicals Europe, Abingdon, UK). Recombinant human CXCL4L1 (PF4var1) as well as the CXCL4 (PF4) were purchased from ChromaTec (Greifswald, Germany). The other recombinant human chemokines were obtained from Peprotech (Hamburg, Germany). All other reagents and chemicals were purchased from Merck KGaA (Darmstadt, Germany), Carl Roth GmbH (Karslruhe, Germany), or Sigma-Aldrich and were of the highest purity degree available.

\section{Cell culture and cultivation of mammalian cell lines}

Jurkat T cells were cultured in RPMI1640 medium (Gibco) supplemented with $10 \%$ fetal calf serum (FCS), 1\% penicillin/streptomycin, and 1x non-essential amino acids (NEAAs, Gibco). The human monocytic cell line MonoMac6 (Ziegler-Heitbrock et al, 1988) was cultured in RPMI1640 medium + GlutaMAX (1x), supplemented with 1x NEAAs, 10\% FCS, and 1\% penicillin/streptomycin. HEK293 cells stably transfected with human CXCR4 (HEK293CXCR4) were used at passage 5 and were cultivated in DMEM medium (Gibco), supplemented with 10\% FCS and 1\% penicillin/streptomycin (Gibco), and used for the experiment between passage 6 and 8 . 
Unless stated otherwise, cells were cultivated in a temperature- and humidity-

188

189

190

191

192

193

194

195 controlled incubator at a temperature of $37^{\circ} \mathrm{C}$ and $5 \% \mathrm{CO}_{2}$. FCS from an EU-approved origin was obtained from Invitrogen-Thermo Fisher Scientific and heat-inactivated prior to usage. Other cell culture reagents, media and supplements were bought from Invitrogen-Thermo Fisher Scientific, unless stated otherwise. Cell lines were originally obtained from the German Society for Microorganisms and Cell Cultures (DSMZ, Braunschweig, Germany) or from the American Type Culture Collections (ATCC).

\section{Isolation of primary human $\mathrm{CD4}^{+} \mathrm{T}$ cells}

Primary human CD4-positive $T$ cells were isolated from enriched peripheral blood mononuclear cell (PBMC) fractions using the human $\mathrm{CD}^{+} \mathrm{T}$ cell isolation kit from Miltenyi Biotec (Bergisch Gladbach, Germany) according to the manufacturer's instructions. Cells were cultivated in RPMI1640 medium, supplemented with 10\% FCS, $1 \%$ penicillin/streptomycin, and $1 \times$ NEAAs in a cell culture incubator at $37^{\circ} \mathrm{C}$ and $5 \% \mathrm{CO}_{2}$ and used for functional assays on the next day. PBMC fractions were obtained by apheresis from conical chambers of a Leucoreduction System Chamber sourced from anonymous platelet donations at the Department of Transfusion Medicine, Cell Therapeutics and Hemostaseology of LMU University Hospital. Studies abide by the Declaration of Helsinki principles and were approved by ethics approval 18-104 of the Ethics Committee of LMU Munich, which encompasses the use of anonymized tissue and blood specimens for research purposes.

\section{Isolation of human platelets}

\section{For immunofluorescent stainings}

Human platelets were isolated from blood, freshly drawn from healthy donors, using a syringe containing $1 / 10$ volume of CTAD-buffer $(0.105 \mathrm{M}$ tri-sodium citrate, $10 \mathrm{mM}$ theophylline, $3.7 \mathrm{mM}$ adenosine, $0.198 \mathrm{mM}$ dipyridamole) (Polack et al, 2001). To prevent platelet activation, the blood was supplemented with prostaglandine E1 (Merck KGaA), Apyrase (New England Biolabs GmbH, Frankfurt am Main, Germany), and EGTA (Sigma- 
215 Aldrich). Briefly, platelets were isolated by sequential centrifugation steps, performed at room 216 temperature (RT) with reduced brake settings. Platelet-rich plasma (PRP) was separated 217 from whole blood by centrifugation for $5 \mathrm{~min}$ at $300 \times \mathrm{g}$, diluted with an equal volume of 218 phosphate-buffered saline (PBS), $\mathrm{pH} 7.4$, and centrifuged again for $10 \mathrm{~min}$ at $200 \times \mathrm{g}$ to remove remaining leukocytes. Finally, platelets were sedimented by centrifugation for $10 \mathrm{~min}$ 220 at $400 \times g$.

\section{For functional studies}

222 Washed human platelets were isolated as previously described (Borst et al, 2012) and 223 subsequently used for functional flow chamber or platelet spreading assays.

Mice and preparation and cultivation of primary mixed cortical cultures for the microglia motility assay

$227 \mathrm{CX} 3 \mathrm{CR} 1^{\mathrm{GFP} /+}$ mice, which were originally obtained from the Jackson Laboratories (strain 228 005582; (Niess et al, 2005)), were established on a pure C57BL/6 background and housed under standardized light-dark cycles in a temperature-controlled air-conditioned environment under specific pathogen-free conditions at the Center for Stroke and Dementia Research

231 (CSD), Munich, Germany, with free access to food and water. Animals were sacrificed under anaesthesia with a mixture of midazolam $(5 \mathrm{mg} / \mathrm{mL})$, medetomidine and fentanyl (MMF). Mouse maintenance and experiments were reviewed and overseen by the institutional 234 animal use and care committee of the local authorities (Regierung von Oberbayern, ROB, 235 Germany) and performed in accordance with the procedures provided by the animal 236 protection representative of CSD.

Primary mixed cortical cultures containing CX3CR1GFP/+ microglia were prepared in 238 96-well imaging plates based on a previously established protocol (Gokce \& Sudhof, 2013) 239 from the cortices of 5 newborn pups of the CX3CR $1^{\mathrm{GFP} /+}$ mouse line (postnatal day 0 ) in 240 plating medium, consisting of modified Minimum Essential Medium (MEM without glutamine 241 and phenol red) (Gibco), supplemented with $0.5 \%$ glucose, $0.02 \%$ sodium bicarbonate, $1 \mathrm{x}$ 242 ITS-supplement (Sigma-Aldrich), 2 mM L-glutamine (Gibco), 1\% penicillin/streptomycin, and 
$24310 \%$ FCS. Cultures were incubated in a humidified atmosphere at $37{ }^{\circ} \mathrm{C}$ and $5 \% \mathrm{CO}_{2}$ for 10

244 d. One day after plating, $80 \%$ of the plating medium was replaced with growth medium, 245 prepared from MEM (without glutamine and phenol red) supplemented with $0.5 \%$ glucose,

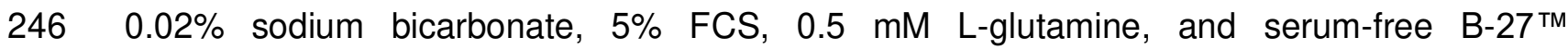
247 supplement (Gibco). On the fourth day after dissection, 50\% of the medium was replaced 248 with growth medium additionally supplemented with $4 \mu \mathrm{M}$ cytosine-1- $\beta$-D-arabinofuranoside 249 (Sigma-Aldrich).

250

\section{Chemokine protein array}

Human chemokines and selected atypical chemokines were spotted on a nitrocellulose membrane at $100 \mathrm{ng}$ per spot and left to dry at RT. Membranes were blocked with $1 \mathrm{x}$ ROTI®Block (Carl Roth) for $2 \mathrm{~h}$ at RT and then probed overnight with biotinylated human MIF (biotin-MIF) at a concentration of $1 \mu \mathrm{g} / \mathrm{mL}$ in either $10 \mathrm{mM}$ Tris- $\mathrm{HCl} \mathrm{pH}, 8.0$ or $10 \mathrm{mM}$ MES, pH 6.0. Subsequently, membranes were washed three times with $0.01 \%$ Tween ${ }^{2} 20$ in water and developed with horseradish-peroxidase (HRP)-conjugated streptavidin (BioTechne GmbH, Wiesbaden-Nordenstadt, Germany), diluted 1:200 in 1x ROTI®Block, for 2 h. After another washing step, bound biotin-MIF was revealed via chemiluminescence using SuperSignal ${ }^{\mathrm{TM}}$ West Pico Chemiluminescent Substrate (Thermo Fisher Scientific) on a LAS3000 Imaging System (Fuji Photo Film Co., LTD., Japan).

\section{Pull-down of CXCL4L1 from cell lysates}

264 MonoMac-6 cells were first washed with PBS and then lysed on ice for 30 min with immunoprecipitation (IP) lysis buffer (1x cell lysis buffer, Cell Signaling, cat\#9803), 100 mM PMSF, and 1x protease and phosphatase inhibitors (ThermoFisher). The purified cell lysates were then incubated with pre-washed streptavidin-conjugated paramagnetic beads

268 (DYNAL $^{\mathrm{TM}}$ Dynabeads $^{\mathrm{TM}}$ M-280 Streptavidin; Invitrogen, cat\#11205D) for $2 \mathrm{~h}$ at $4{ }^{\circ} \mathrm{C}$ 269 (preclearing step). After centrifugation, the supernatant was incubated with biotinylated 270 human MIF by gentle, constant shaking on a rotary shaker overnight at $4{ }^{\circ} \mathrm{C}$. To capture 
271 MIF/CXCL4L1 complexes, prewashed streptavidin-conjugated beads were added to the 272 precleared lysates, and the mixture was incubated for $2 \mathrm{~h}$ at $4{ }^{\circ} \mathrm{C}$ on a rotary shaker. Beads 273 were separated from the lysate using a magnetic stand (Dynal ${ }^{\mathrm{TM}}$ MCP-S) and washed three 274 times with lysis buffer. The supernatant was removed and the beads were resuspended in 275 LDS sample buffer (Invitrogen) and boiled at $95{ }^{\circ} \mathrm{C}$ for $15 \mathrm{~min}$. Samples were subjected to 276 SDS-PAGE and analyzed by Western blotting. For this purpose, equal amounts of protein 277 were loaded onto $11 \%$ SDS-polyacrylamide gels (NuPAGE, Thermofisher) and transferred to 278 polyvinylidene difluoride (PVDF) membranes (Carl Roth, Karlsruhe, Germany). Membranes were blocked in PBS-Tween-20 containing 5\% BSA for $1 \mathrm{~h}$ and incubated overnight at $4{ }^{\circ} \mathrm{C}$ with rabbit polyclonal anti-MIF antibody Ka565 (Bernhagen et al., 2007) or rabbit polyclonal anti-PF4V1 IgG PA5-21944 (Invitrogen) diluted in blocking buffer. Proteins were revealed using anti-rabbit HRP as a secondary antibody. Signals were detected by chemilumi283 nescence on an Odyssey® Fc Imager (LI-COR Biosciences GmbH, Bad Homburg, 284 Germany) using SuperSignal ${ }^{\mathrm{TM}}$ West Dura ECL substrate from ThermoFisher Scientific and specific primary antibodies as indicated.

\section{CelluSpot peptide array}

288 The CelluSpot peptide array method has been described previously (Lacy et al., 2018). 289 Briefly, 15-meric peptides, positionally frame-shifted by three residues and spanning the 290 entire sequence of CXCL4 and CXCL4L1, were synthesized on modified cellulose disks 291 (Intavis MultiPep RSi/CelluSpot Array, Cologne, Germany). Peptides were then further 292 processed by dissolving the cellulose, and spotted on coated glass slides using a slide spotting robot from Intavis. Slides were incubated in blocking buffer (50 mM Tris-buffered saline, $\mathrm{pH} 7.4,1 \% \mathrm{BSA}, 0.1 \%$ Tween® 20 ), washed (50 mM Tris-buffered saline, $\mathrm{pH} 7.4$, $0.1 \%$ Tween $\left.{ }^{\circledR} 20\right)$ and probed with biotinylated human MIF (3 $\mu \mathrm{M}$ in blocking buffer). After 296 washing, slides were developed with a dilution of streptavidin-conjugated horseradish 297 peroxidase (Roche) in blocking buffer. Bound MIF was revealed by chemiluminescence on 298 an Odyssey ${ }^{\circledR}$ FC imager using the SuperSignal ${ }^{\mathrm{TM}}$ West Dura ECL substrate 


\section{Microscale thermophoresis (MST)}

300 Protein-protein interactions were analyzed via microscale thermophoresis on a Monolith 301 NT.115 instrument equipped with green/red filters (NanoTemper Technologies, Munich, 302 Germany). Measurements were performed at $25^{\circ} \mathrm{C}$ at both $40 \%$ and $80 \% \mathrm{MST}$ power. LED 303 excitation power was adjusted to 90 or $95 \%$ in order to obtain an initial fluorescence count of 304700 to 800 . MST traces were recorded for $40 \mathrm{~s}(-5 \mathrm{~s}$ to $+35 \mathrm{~s})$, according to default settings 305 with the sample being heated from 0 to $30 \mathrm{~s}$. All measurements were performed in assay 306 buffer (10 mM Tris-HCl, pH 8.0, 0.01\% BSA). MST-Red-MIF was used at a fixed 307 concentration, mixed 1:1 with serial dilutions of either CXCL4 (Peprotech, Hamburg, 308 Germany) or CXCL4L1 (ChromaTec, Greifswald, Germany) (final MIF concentrations: 456 $309 \mathrm{nM}$ or $312 \mathrm{nM}$, respectively). Prior to measurement, the prepared samples were incubated 310 for at least 30 min on ice. MST traces of multiple experiments were analyzed according to 311 the $K_{D}$ model using the default $T$-jump settings, focusing on the temperature related intensity 312 change (TRIC) of the fluorescent label ("cold region" from -1 to $0 \mathrm{~s}$, "hot region" from 0.5 to $3131.5 \mathrm{~s}$ ) using the MO.AffinityAnalysis V2.3 software (NanoTemper Technologies). Curve fitting 314 for data presentation was performed by GraphPad Prism Version 6.07 ('one site - total 315 binding').

317 Analysis of protein-protein interactions by surface plasmon resonance (SPR)

318 Surface plasmon resonance measurements were performed using a Biacore X100 319 instrument (GE Healthcare Europe $\mathrm{GmbH}$ ) and neutravidin-modified $\mathrm{C} 1$ sensor chips. Biotin320 MIF was immobilized on flow cells to 1064.8 RU. CXCL4 (Peprotech, Hamburg, Germany) 321 and CXCL4L1 (ChromaTec, Greifswald, Germany), used at concentrations in the range of 3220.125 to $20 \mu \mathrm{g} / \mathrm{mL}$ in running buffer (HBS-EP+ Buffer: $0.01 \mathrm{M}$ HEPES, $0.15 \mathrm{M} \mathrm{NaCl}, 0.003 \mathrm{M}$ 323 EDTA and $0.05 \% \mathrm{v} / \mathrm{v}$ surfactant P20) were injected at a flow rate of $60 \mu \mathrm{L} / \mathrm{min}$. The complex 324 was allowed to associate and dissociate for $90 \mathrm{~s}$ and $240 \mathrm{~s}$, respectively. Surfaces were 325 regenerated with 2 pulses $(60 \mathrm{~s})$ of $30 \mathrm{mM} \mathrm{NaOH}$ and $2 \mathrm{M} \mathrm{NaCl}$. Responses from analyte 
326 injections were fitted to a 1:1 Langmuir interaction profile using Biacore $\mathrm{X} 100$ evaluation

327 2.0.1 Plus package software.

\section{Transwell migration assay}

330 Transwell migration experiments to study the influence of CXCL4L1 on MIF-mediated chemotaxis responses were performed with Jurkat T cells. Briefly, Jurkat cells were diluted in

332 RPMI1640 medium at a density of $1 \times 10^{7}$ cells $/ \mathrm{mL}$. Cells were placed in the upper chamber of a 24-well Transwell insert with $5 \mu \mathrm{m}$ pore size (Corning, Kaiserslautern, Germany). $16 \mathrm{nM}$ MIF, either alone or pre-incubated (30 min on ice to allow for complex formation) with $32 \mathrm{nM}$ of CXCL4L1, as well as $32 \mathrm{nM} \mathrm{CXCL4L1} \mathrm{alone} \mathrm{were} \mathrm{added} \mathrm{to} \mathrm{the} \mathrm{lower} \mathrm{chamber} \mathrm{as} \mathrm{a}$ chemoattractant. After a $12 \mathrm{~h}$ migration interval at $37{ }^{\circ} \mathrm{C}$ and $5 \% \mathrm{CO}_{2}$, migrated cells were recovered from the lower chamber and counted via flow cytometry by using CountBright ${ }^{\mathrm{TM}}$ absolute counting beads (Molecular Probes-Invitrogen). In a similar experimental setup, the influence of CXCL4 on MIF-mediated chemotaxis was tested as well. MIF was used at a concentration of $16 \mathrm{nM}$ and CXCL4 (ChromaTec, Greifswald, Germany) at 32 nM.

\section{D migration of human $\mathrm{CD}^{+} \mathrm{T}$ cells}

343 The migratory behavior of primary human T cells was assessed by three-dimensional (3D) 344 migration methodology using time-lapse microscopy and single cell tracking using the 3D 345 chemotaxis $\mu$-Slide system from Ibidi GmbH (Munich, Germany). The method was performed 346 following a slight modification of the established Ibidi dendritic cell protocol for human 347 monocytes, as described previously (Kontos et al., 2020). Briefly, isolated CD4+ human T 348 cells $\left(3.5 \times 10^{6}\right)$ were seeded in a rat tail collagen type-I gel (Ibidi, Munich, Germany) in 349 DMEM and subjected to a gradient of human MIF, CXCL4L1, or a pre-incubated combination of both. Cell motility was monitored performing time-lapse imaging every 0.5 or 2 min at 37

$351{ }^{\circ} \mathrm{C}$ for a period of 120 min to cover either a short or extended migration period, using a Leica 352 DMi8 inverted microscope (Leica Microsystems, Wetzlar, Germany) and Leica live cell353 imaging software (LAS X version 3.7.4). Images were imported as stacks to ImageJ version 
$3541.51 \mathrm{n}$ and analyzed with the manual tracking and Chemotaxis and Migration tool (Ibidi $355 \mathrm{GmbH})$ plugin for ImageJ.

\section{Motility measurement of primary murine microglia}

358 The motility of mouse microglia was determined using mixed cortical cultures, established 359 and cultivated as stated above. A day prior to imaging, the medium was changed to 360 Hibernate A medium (Gibco) in order to maintain a physiological $\mathrm{pH}$ value during imaging. 361 Prior to imaging, different wells of cells from each individual pup were treated with either 8 $362 \mathrm{nM}$ MIF, $1.6 \mathrm{nM} \mathrm{CXCL4L1}$, or both (pre-incubated for $30 \mathrm{~min}$ on ice to allow for complex 363 formation). A control group was treated with $20 \mathrm{mM}$ sodium phosphate buffer, $\mathrm{pH}$ 7.4. Cell 364 motility was monitored by time-lapse imaging for $15 \mathrm{~h}$ at $37^{\circ} \mathrm{C}$ with recordings every $5 \mathrm{~min}$, using a Leica DMi8 inverted Life Cell Imaging System using the FITC channel for visualizing

366 the GFP-positive cells. Images were imported as stacks to ImageJ software version 1.51n 367 and analyzed with the manual tracking and Chemotaxis and Migration tool Plugin for ImageJ 368 from Ibidi. In order to quantify microglial motility from the time-lapse videos, 20-25 GFP369 positive microglia per treatment group were randomly selected and manually tracked 370 throughout all frames. Cells that died or moved out of the frame were excluded from the 371 analysis. Accumulated distance of each tracked microglia was calculated with Chemotaxis 372 and Migration Tool (Ibidi).

\section{$374 \quad$ Fluorescence polarization spectroscopy}

375 Fluorescence polarization was measured using a JASCO FP-6500 fluorescence 376 spectrophotometer equipped with FDP-223 and FDP-243 manual polarizers (JASCO 377 Deutschland $\mathrm{GmbH}$, Pfungstadt, Germany). Preparation of stock solutions, measurements and analysis were performed essentially following a previously published protocol (Kontos et 379 al., 2020). For binding/inhibition experiments, mixtures of Alexa 488-labeled MIF (10 nM) in 380 the absence/presence of CXCL4L1 (1.6 $\mu \mathrm{M}$ ) (or $20 \mathrm{mM}$ sodium phosphate buffer, $\mathrm{pH} 7.2$ ), 381 and non-labeled msR4M-L1 peptide (concentration between $1 \mathrm{nM}$ to $10 \mu \mathrm{M}$ ) were prepared 
382 in $10 \mathrm{mM}$ sodium phosphate, $\mathrm{pH} 7.2$, containing $2 \%$ hexafluoro-isopropanol (HFIP). Where 383 CXCL4L1 was added as a putative inhibitor, Alexa488-MIF and CXCL4L1 were mixed and 384 incubated for 30 min prior to measurements. Bandwidth for excitation and emission was set 385 at $5 \mathrm{~nm}$ and time response at $0.5 \mathrm{~s}$. The excitation wavelength was $492 \mathrm{~nm}$ and emission was recorded at $519 \mathrm{~nm}$. Measurements were taken at RT within 2 to $3 \mathrm{~min}$ upon preparation of the solutions. Polarization $P$ was calculated according to the equation $P=\left(I_{\|}-G \cdot I_{\perp}\right) /\left(I_{\|}+\right.$

$388 \mathrm{G} \cdot I_{\perp}$ ), with $I_{\|}$as the intensity of emitted light polarized parallel to the excitation light and $I_{\perp}$ as 389 the intensity of emitted light polarized perpendicular to the excitation light. The $G$ factor was 390 calculated based on the instrumental documentation (Moerke, 2009). Apparent $K_{D}$ values were calculated assuming a 1:1 binding model (Yan et al, 2006), using sigmoidal curve fitting with OriginPro 2016 (OriginLab Corporation, Northampton, MA, USA).

\section{Label-free dynamic mass redistribution (DMR) assay}

395 Analysis of dynamic mass redistribution of adherent cells was performed on an EnSpire Multimode plate reader equipped with an Epic® label-free measurement module 397 (PerkinEImer Inc., Waltham MA, USA) according to the manufacturer's instructions for cellbased label-free DMR measurements (Schroder et al, 2010). The assay protocol was adapted according to a previous publication to be performed in EnSpire label-free 96-well fibronectin-coated cell assay microplates (Corning $\mathrm{GmbH}$, Amsterdam, The Netherlands) with HEK293 cells stably expressing human CXCR4 (Krammer et al., 2021). Briefly, 40.000 402 cells were seeded into each well and cultivated overnight $\left(37^{\circ} \mathrm{C}, 5 \% \mathrm{CO}_{2}\right)$ to achieve a confluency of $>70 \%$. Prior to the assay, the medium was exchanged with DMR assay buffer

404 (20 mM HEPES and 1\% DMSO in HBSS, $\mathrm{pH}$ 7.4) and the assay plate left for $6 \mathrm{~h}$ to equilibrate to ambient temperature. Baseline measurements for each well were recorded for $10 \mathrm{~min}$ every $30 \mathrm{~s}$ prior to treatment of the cells with chemokines, inhibitors, or the corresponding buffers as control. Treatments were applied to each well as a $5 x$ concentrated 408 stock, prepared in assay buffer. For treatment with MIF/CXCL4L1 complexes, both proteins 409 were mixed in assay buffer and incubated for $5 \mathrm{~h}$ at RT. Directly after addition of the stimuli, 
410 the DMR response was recorded for the indicated duration. The DMR response resembles

411 the wavelength shift of the light reflected from the sensor integrated in the assay microplates

412 and serves as a cumulative cellular response signal. Measurements were performed on two 413 replicates per treatment and results are presented as their mean value.

414

415 Staining of human thrombus specimens

416 Human thrombus tissue specimens, obtained as disposable material from vascular surgery 417 procedures (ethics allowance LMU Munich 18-104 and TUM-MRI project \# 2799/10), were 418 embedded in Tissue Tek O.C.T. Compound (Sakura Finetek Germany GmbH, Staufen, 419 Germany), frozen, and cut into $5 \mu \mathrm{m}$ sections using a CM 1950 Cryostat (Leica Biosystems). 420 The cryosections were transferred to microscopy slides and stored at $-80^{\circ} \mathrm{C}$ until use.

421 Hematoxylin \& eosin (HE) staining of thrombus sections

422 Cryosections of human thrombus tissue were stained with Mayer's hematoxylin and eosin 423 (HE) according to standard protocols. Briefly, after thawing and brief air drying and 424 rehydration, sections were incubated in Mayer's hematoxylin solution (Sigma-Aldrich) for 15 425 min. After thorough rinsing of the samples, $0.5 \%$ eosin $Y$ solution (Sigma-Aldrich) was 426 applied as a counterstain for $30 \mathrm{~s}$. After dehydration of the tissue in $95 \%$ ethanol, $100 \%$ ethanol (Merck KGaA) and xylene (VWR International $\mathrm{GmbH}$ ), the sample was covered with resinous mounting medium (Eukitt, Sigma-Aldrich), covered with a glass coverslip and examined by light microscopy (Leica Dmi8 inverted microscope using a DMC2900 digital camera (Leica Microsystems; 10x objective).

\section{Staining of platelets}

433 Freshly isolated platelets were fixed with 4\% paraformaldehyde (PFA) in PBS (Morphisto $434 \mathrm{GmbH}$, Frankfurt a. M., Germany) for 10 min and subsequently permeabilized using 1x Perm 435 buffer (Invitrogen) for 15 min. After washing, platelets were blocked in ROTI®Block (Carl 436 Roth) for $1 \mathrm{~h}$. Immunofluorescent staining was performed as described above for the human 437 thrombus specimens, except that the anti-human MIF antibody was used at a dilution of 1:20 
438 and the anti-human CXCL4L1 antibody at a dilution of 1:50. Stained platelets were then 439 washed in blocking buffer and mounted on poly-L-ornithine-coated glass slides using 440 ProLong ${ }^{\mathrm{TM}}$ Glass Antifade mountant (Invitrogen), covered with coverslips and stored at $4{ }^{\circ} \mathrm{C}$ 441 until imaging by multiphoton microscopy.

443 Multiphoton laser-scanning microscopy (MPM) and FLIM-FRET

444 Imaging was conducted using a multispectral TCS SP8 DIVE FALCON LIGHTNING 445 microscope (Leica, Germany) equipped with filter-free 4TUNE NDD detection module, an 446 extended IR spectrum tunable laser (New InSight ${ }^{\circledR}$ X3 $^{\text {тм }}$, Spectra-Physics) $(680-1300 \mathrm{~nm})$ 447 and fixed IR laser (1045 nm), advanced Vario Beam Expander (VBE), Ultra-high-speed 448 resonance scanner $(8 \mathrm{kHz}), \mathrm{HC}$ PL IRAPO 25x/1.0 WATER objective, and FLIM-FRET 449 modality. Images were collected in a sequential scanning mode using hybrid diode detectors 450 Reflected Light Hybrid Detectors (HyD-RLD) (Alexa Fluor-488: excitation 965 nm / emission 451 479-568 nm; Cy3: excitation $1095 \mathrm{~nm}$ / emission 538-650 nm) and were handled using the 452 LAS-X software package. Deconvolution microscopy was performed using the Leica 453 LIGHTNING (adaptive deconvolution) application.

454 For fluorescence lifetime imaging (FLIM) and FLIM-FRET measurements, up to 1000 455 photons per pixel were captured in a time-correlated single photon counting (TCSPC) mode. 456 Fluorescence lifetime decay data were fitted using Leica FALCON (FAstLifetime CONtrast) 457 software. The fitting was assessed by randomly distributed residuals and by low Chi-square $458\left(X^{2}\right)$ values. The number of components used for the fittings was manually fixed to a value $459(n=2-3)$ to minimize $x^{2}$ values. The fluorescence lifetime of the donor was acquired similarly 460 in the absence of the acceptor. 


\section{Proximity ligation assay (PLA)}

464 For detection of protein complexes by proximity ligation assay (PLA), the Duolink ${ }^{\mathrm{TM}}$ InSitu 465 Orange Starter Kit Mouse/Rabbit (DUO92102) from Sigma Aldrich was used. Following 466 scouting experiments to establish the PLA methodology in thrombus material, cryosections of 467 the thrombi were prepared by treatment with cold acetone for 6 min at $4{ }^{\circ} \mathrm{C}$ and for $30 \mathrm{~min}$ at 468 RT. Samples were rehydrated in PBS for $10 \mathrm{~min}$ and hydrophobic barriers were applied to 469 the microscopy slide using an ImmoEdgeTM Pen (Vector Laboratories Inc. Burlingame, 470 USA).

471 For PLA detection, the Duolink $\AA$ PLA Fluorescence protocol provided by the manufacturer 472 was essentially followed, using primary antibodies against human MIF (mouse anti-MIF D2, 473 sc-271631, Santa Cruz Biotechnology Inc., Dallas, USA; 1:20) and against human CXCL4L1 474 (rabbit anti-CXCL4L1, PA5-21944, Invitrogen; 1:50). Samples were then prepared for 475 microscopy using Duolink® mounting medium with DAPI, and coverslips sealed with 476 commercially available nail polish and stored at $4^{\circ} \mathrm{C}$ until imaging by confocal microscopy 477 using a Zeiss LSM880 AiryScan microscope was performed.

\section{$479 \quad$ Flow chamber assay with platelets}

480 Chemokines were diluted in calcium-free PBS, $\mathrm{pH} 7.4$, at their final concentrations (MIF: 16 $481 \mathrm{nM}$; CXCL4L1: $32 \mathrm{nM}$ ) and allocated into separate reaction tubes. $200 \mu \mathrm{L}$ of each solution 482 were distributed onto separate collagen-coated cover slips (100 $\mu \mathrm{g} / \mathrm{mL})$ and incubated for 2 483 h. Cover slips were blocked with PBS, $\mathrm{pH} 7.4$, containing 1\% BSA for $1 \mathrm{~h}$. Next, human 484 whole-blood was diluted at a 5:1 ratio with PBS, $\mathrm{pH} 7.4$, containing calcium. Before perfusion, the blood was incubated with fluorochrome 3,3'-dihexyloxacarbocyanine iodide 486 ( $\mathrm{DiOC}_{6}, 1 \mathrm{mM}$; Sigma Aldrich) for $10 \mathrm{~min}$ at RT. Thereafter, the blood was allocated into 1 $\mathrm{mL}$ syringes and perfused over the different cover slips, through a transparent flow chamber 488 with high shear rate $\left(1000 \mathrm{~s}^{-1}\right)$ for $5 \mathrm{~min}$. Per run, one 2-minute video clip was recorded (200 489 ms/frame, Nikon Eclipse Ti2-A, 20x objective). Afterwards, the chamber was rinsed and 490 pictures were taken of five representative areas using the same objective. The covered area 
491

492

493

494

495

496

497

498

499

500

501

502

503

504

505

506

507

508

509

510

511

512

513

514

515 To simulate the interaction of monomeric MIF with CXCL4 and CXCL4L1 in their monomeric 516 forms, rigid protein-protein docking, followed by clustering of the 1000 lowest energy 517 structures and removal of steric clashes was performed using the ClusPro 2.0 webserver,

was analyzed using the NIS-Elements AR software (Nikon) and the mean percentage of the covered area, the mean thrombus area as well as the mean thrombus count were determined.

\section{Platelet spreading analysis}

Fibrinogen-coated (100 $\mu \mathrm{g} / \mathrm{mL}$, Sigma Aldrich) coverslips were preincubated with MIF (16 nM), CXCL4L1 (32 nM), or MIF (16 nM) and CXCL4L1 (32 nM) together, for $2 \mathrm{~h}$. Afterwards, isolated human platelets were diluted in Tyrodes buffer $(\mathrm{pH} 7.4)$ to match a concentration of 15.000 cells $/ \mu \mathrm{L}$. Platelets were supplemented with $1 \mathrm{mM} \mathrm{CaCl}{ }_{2}$, activated with $1 \mu \mathrm{g} / \mathrm{mL} \mathrm{CRP}$ $\mathrm{XL}$ (CambCol, Cambridge, UK), and incubated on the previously prepared fibrinogen-coated coverslips for 30 or 60 min at RT. Thereafter, platelets were fixed with $4 \%$ formaldehyde (Sigma Aldrich) for $10 \mathrm{~min}$, and washed three times with PBS, $\mathrm{pH}$ 7.4. The coverslips were mounted onto slides and five images from randomly selected areas were taken using a Nikon Eclipse Ti2-A microscope with a 100x DIC objective. Subsequently a quarter of each image with at least 20 cells was analyzed.

\section{Protein structure visualization}

Three-dimensional structures as well as the surface charge distribution of human MIF, CXCL4 and CXCL4L1 were visualized using the PyMOL Molecular Graphics System software, version 1.8.2.2 (Schrödinger, LLC). The structures represent the Protein Data Bank (PDB) files for MIF (PDB ID: 3DJH), CXCL4 (PDB ID: 1F9Q), and CXCL4L1 (PDB ID: 4HSV), orour molecular docking results.

\section{Protein-protein docking}


518 with single chains of MIF and CXCL4L1 defined as 'receptor' and 'ligand', respectively 519 (Kozakov et al, 2017; Vajda et al, 2017).

520

\section{Statistical analysis}

522 Statistical analysis was performed using GraphPad Prism Version 6.07 software. Unless 523 stated otherwise, data are represented as means \pm standard deviation (SD). After testing for 524 normality, data were analyzed either by two-tailed Student's T-test, Mann-Whitney U test, or 525 Kruskal-Wallis test as appropriate. Differences with $P<0.05$ were considered to be 526 statistically significant.

527 
Results

3 High affinity binding between the atypical chemokine MIF and the platelet CXC chemokine CXCL4L1

To begin to test the hypothesis that chemokine-chemokine interactions may extend to ACKs and that MIF may form heterocomplexes with classical chemokines, we applied unbiased chemokine protein array technology (Figure $1 A-B$ ), as previously successfully used to map formation of heterocomplexes between different classical chemokines (von Hundelshausen et al., 2017). In addition to 47 human chemokines covering all four sub-classes (CXC-, CC-, CX3C- and C-type CKs) we also included structurally related and positively charged protein mediators including ACKs/DAMPs such as HMGB1, HBDs, and peroxiredoxins (Prxs) (Shichita et al, 2012; He et al, 2019), as well as MIF itself and the MIF homolog Ddopachrome tautomerase (D-DT)/MIF-2 as spotted proteins in the protein array. Probing of the array with biotin-conjugated MIF and streptavidin-POD (StrAv-POD) revealed highintensity spots indicative of a tight interaction of MIF with CXCL4L1 and Prx1 (Figure 1B-C). Weaker spots were detected for CCL28, CXCL9, Prx6, and MIF itself. No spot intensity whatsoever was observed for any of the other immobilized proteins, indicating that none of the other 44 chemokines interacts with MIF. This also included CXCL8 and CXCL12, which share their receptors CXCR2 and CXCR4, respectively, with MIF (Bernhagen et al., 2007).

Similarly, no binding signal of biotin-MIF was detected with HMGB1, a DAMP which has been demonstrated to form heterodimers with CXCL12 and for which a functional interaction with MIF has been suggested (Ma et al, 2017; Schiraldi et al., 2012), nor for the human $\beta$-defensins HBD1 or HBD2 (Figure 1B-C). Importantly, when testing a control chemokine array developed with StrAv-POD without a biotin MIF incubation step, only one signal was not fully specific. This was the signal for Prx1 so that its interpretation was not possible (Supplementary Figure 1A). Biotin-MIF also bound to MIF itself, but not to MIF-2 
1 reported to form higher-order hexameric complexes (Bai et al, 2012), this result further 2 verified the validity of the chemokine array approach for MIF.

A striking observation was that biotin-MIF specifically interacted with the immobilized platelet chemokine CXCL4L1, but not with CXCL4 (Figure $1 \mathrm{C}$ and Supplementary Figure 1B). CXCL4 and CXCL4L1 are highly homologous chemokines, their sequences only differ by three amino acids, and CXCL4L1 has also been suggested to be a decoy chemokine paralog of CXCL4. Given this remarkable specificity of the interaction with MIF and that the spot corresponding to biotin-MIF and CXCL4L1 was the strongest interaction detected on the array, we focused on CXCL4L1 as a novel candidate interactor of MIF.

We first verified the interaction by co-immunoprecipitation using whole cell lysates of MonoMac6 cells, which we found to express substantial amounts of CXCL4L1. Semiendogenous pulldown of proteins from MonoMac6 lysates by biotin-MIF and StrAv magnetic beads and Western blot using an anti-human CXCL4L1 antibody revealed a specific band for CXCL4L1, which was absent when the pulldown was performed without biotin-MIF preincubation (Figure $2 A$ ). Pulldown specificity was further confirmed by Western blot against MIF. We next applied surface plasmon resonance ('Biacore') methodology, which was previously successfully used to characterize interactions within the classical chemokine interactome (von Hundelshausen et al., 2017). To study the MIF/CXCL4L1 interaction, MIF chips were exposed to increasing concentrations of CXCL4L1 in the soluble phase. The obtained surface plasmon resonance response curves indicated that MIF specifically binds to CXCL4L1 (Figure 2B-C). Quantitative analysis determined a $\mathrm{K}_{\mathrm{D}}$ value of $116 \pm 16 \mathrm{nM}$ (mean $\pm \mathrm{SD}$ ) indicating high-affinity binding between MIF and CXCL4L1. By contrast, no appreciable signal was detectable for the incubation with increasing concentrations of CXCL4 and no $K_{D}$ could be derived, verifying the specificity of the MIF/CXCL4L1 interaction in this set-up. To further confirm the MIF/CXCL4L1 interaction, we next applied microscale thermophoresis (MST), which relied on the interaction between MST-Red-labeled MIF and its

27 binding partner, with both partners in the soluble phase. This methodology was recently established for MIF (Kontos et al., 2020). MST titrations of MST-Red-MIF with increasing 
concentrations of CXCL4L1 revealed a typical sigmoidal binding curve with a derived binding constant $\left(\mathrm{K}_{\mathrm{D}}=159.8 \pm 16.8 \mathrm{nM}\right)$ that was similar to that obtained by surface plasmon resonance (Figure 2D-E). In contrast, binding was much weaker when CXCL4 was titrated and accordingly a low affinity $K_{D}$ in the micromolar range was determined $\left(K_{D}=2.0 \pm 0.8\right.$ $\mu \mathrm{M})$.

Heterodimer formation between classical chemokines relies on CC-type or CXC-type interactions. To determine which residues in CXCL4L1 are critical for the interaction with MIF, we employed peptide array technology. A set of 15-meric peptides derived from the CXCL4L1 sequence, positionally frame-shifted by three amino acids to cover the entire sequence of the processed chemokine, were synthesized and immobilized on glass slides and arrays, and probed with biotin-MIF. The most pronounced binding signal was observed for peptides representing the sequence region, which corresponds to the $\beta 2$-strand motif IKAGPHCPTAQLIAT of CXCL4L1 (Supplementary Figure 2A-B). A second peak encompasses the N-terminal sequence QCLCVKTTSQVRPRH. The difference in the 3D structures of CXCL4 and CXCL4L1 is characterized by a significant conformational rearrangement of the $\alpha$-helix (Kuo et al, 2013), although the sequence of CXCL4 differs from that of CXCL4L1 in only three $\alpha$-helical residues (L58P, K66E, L67H with the conformational difference being mainly governed by the L67H exchange). In this respect, CXCL4 showed an essentially identical peptide binding profile as that of CXCL4L1 at the N-terminus as expected, but a slightly different pattern at the $\beta 2$ strand region GPHCPTAQLIATLKN, that is packed onto the C-terminal a-helix (Supplementary Figure 2A-B). Peptide array-based mapping of the CXCL4L1 residues involved in MIF binding was confirmed by molecular docking simulations. Docking applying the ClusPro software predicted that the $\beta$-sheet region including the IKAGPHCPTAQLIAT motif is located near the MIF contact site, facing the 4stranded $\beta$-sheet of a single MIF monomer chain. This interaction could be promoted by an energetically favorable complementary electrostatic interaction between the two surfaces (Supplementary Figure 2C). 
Together, the co-immunoprecipitation, Biacore, and MST studies confirmed specific binding between MIF and CXCL4L1 and determined a high-affinity binding constant in the 100-150 nM range for the interaction. Analysis of the binding interface by peptide arraybased mapping and molecular docking provides an initial prediction of the residues involved in the CXCL4L1/MIF binding site.

6

\section{MIF/CXCL4L1 heterocomplex formation attenuates MIF-mediated inflammatory/athero-} genic activities

We next wished to determine a potential functional role of MIF/CXCL4L1 heterocomplex formation. CXCL4L1 is a potent angiostatic chemokine acting through CXCR3 (Struyf et al, 2011), but its role in inflammatory responses and atherogenesis is not well understood. Proatherogenic activities of MIF have been extensively characterized and are mainly mediated through non-cognate interaction of MIF with CXCR2 and CXCR4 (Bernhagen et al., 2007; Sinitski et al., 2019). Here, we hypothesized that MIF/CXCL4L1 complex formation could predominantly influence CXCR4-mediated pathways of MIF.

We first asked whether MIF-elicited T-cell chemotaxis, a well-characterized atherogenic MIF effect mediated via T-cell-expressed CXCR4 (Bernhagen et al., 2007), is affected by CXCL4L1. Scouting experiments using Jurkat T-cells confirmed that, when added to the lower chamber of a Transwell migration device as a chemotattractant, MIF elicited chemotaxis with a chemotactic index (CTX) of approximately 2. Moreover, when CXCL4L1 was preincubated with MIF to allow for complex formation, no upregulation of Jurkat T-cell chemotaxis was observed, while CXCL4L1 alone exhibited neither a chemotactic nor inhibitory effect (Supplementary Figure $3 A$ ). In line with the observed lack of binding between MIF and CXCL4, MIF-mediated Jurkat T-cell chemotaxis was not attenuated by coincubation with CXCL4, which by itself did not significantly enhance Jurkat T-cell chemotaxis (Supplementary Figure 3B). To test the physiological relevance of this finding, we next studied primary $\mathrm{CD}^{+}{ }^{+} \mathrm{T}$-cell chemotaxis and also applied a three-dimensional migration setup, following individual cell migration trajectories by live cell imaging. MIF potently triggered 
1 T-cell migration as evidenced by a significant increase in forward migration index (FMI)

2 (Figure $3 A-B)$, confirming previous data showing CXCR4-dependent stimulation of monocyte

3 migration by MIF (Kontos et al., 2020). This effect was abrogated when MIF was coincubated

4 with CXCL4L1, while CXCL4L1 alone had no effect on 3D T-cell motility. This suggested that MIF/CXCL4L1 heterocomplex formation interferes with MIF/CXCR4-stimulated chemotaxis of

6 T cells.

To study the potential relevance of these findings for other inflammatory/immune cell types, we next evaluated the effect of MIF/CXCL4L1 complex formation on microglial motility in the physiological setting of cortical brain cultures. MIF promotes the motility of Egfp ${ }^{+}$ microglia in murine cortical brain cultures ex vivo in a Cxcr4-dependent manner, as read out by live microscopy and as indicated by blockade of the MIF effect by the soluble CXCR4 mimicking peptide msR4M-L1(Supplementary Figure 3C). Importantly, MIF-triggered microglia migration in this setting was fully ablated when CXCL4L1 was added together with MIF following preincubation, while CXCL4L1 alone had no effect on microglia motility (Figure $3 C-D)$. This indicated that CXCL4L1/MIF heterocomplex formation attenuates MIF's CXCR4dependent effect on microglia migration.

\section{MIF/CXCL4L1 heterocomplex formation inhibits MIF binding to CXCR4}

The cell migration experiments implied, but did not directly test, the notion that MIF/CXCL4L1 complex formation affects MIF signaling through the CXCR4 pathway. To test the involvement of CXCR4 directly, we performed a binding competition experiment that capitalized on our recent identification of a MIF-binding CXCR4 ectodomain-mimicking peptide msR4M-L1 (Kontos et al., 2020). Employing fluorescence polarization spectroscopy (FP), titration of increasing concentrations of msR4M-L1 with Alexa 488-MIF led to a pronounced sigmoidal change in the FP signal (Figure 4A), in line with previous data showing high affinity binding between MIF and msR4M-L1 (Kontos et al., 2020). By contrast, when Alexa 488-MIF was preincubated with CXCL4L1 before the titration, the FP signal was 
ablated (Figure 4A), suggesting that MIF/CXCL4L1 heterocomplex formation interfered with MIF binding to the CXCR4 mimic.

To further confirm an interference of heterocomplex formation with the MIF/CXCR4 pathway, we next studied dynamic mass redistribution (DMR) responses in HEK293 cells stably transfected with human CXCR4. Incubation of HEK293-CXCR4 transfectants with MIF but not control buffer led to a pronounced time-dependent increase in the DMR signal as a real-time readout of an integrated cellular response of living HEK293 cell activation through the MIF/CXCR4 receptor signaling pathway (Figure 4C). This signal was markedly attenuated by the small molecule CXCR4 inhibitor AMD3100, whereas the DMR curve of AMD3100 alone was similar to the control buffer curve, confirming CXCR4-dependency of the MIF-induced signal. Of note, preincubation of MIF with CXCL4L1 led to an appreciable reduction in the DMR response curve as well, when compared to cell stimulation with MIF alone, while CXCL4L1 alone and buffer control showed no effect (Figure 4C).

Together, the competition binding study and the DMR experiment confirmed the notion that complexation by CXCL4L1 interferes with binding of MIF to CXCR4 and its ability to activate CXCR4-mediated cell responses.

MIF and CXCL4L1 colocalize and form complexes in human platelet aggregates and

\section{clinical thrombus specimens}

CXCL4L1 is an abundant platelet chemokine (Karshovska et al, 2013; von Hundelshausen et al, 2007) and we previously found that platelets also are a rich source of MIF (Strüßmann et al, 2013). The colocalization of MIF and CXCL4L1 in sub-cellular platelet compartments has not yet been studied, but a cell biological characterization of CXCL4 suggested that this paralog may be localized in a different intracellular platelet compartment than MIF (Strüßmann et al., 2013). Notwithstanding, we surmised that colocalization and complex formation between MIF and CXCL4L1 may occur extracellularly after secretion from activated platelets. 
Initial evidence for a colocalization of CXCL4L1 and MIF following co-secretion from activated platelets came from human platelet preparations that aggregated due to handling stress. Examination of these aggregates by multi-photon microscopy (MPM) using an Alexa 488 signal to label MIF and Cy3 immunofluorescence for CXCL4L1 revealed several areas with an apparent colocalization of MIF and CXCL4L1 (Figure 5A). Colocalization was also detectable in areas with more isolated non-aggregated platelets (Figure 5B). These areas were then subjected to an in-depth analysis by fluorescence lifetime imaging-Förster resonance energy transfer (FLIM-FRET) capitalizing on the Alexa 488/Cy3 FRET donorlacceptor pair. For molecule-molecule interactions within a distance range of 1-10 nm, FLIMFRET monitors the change in fluorescence lifetime of the donor via FRET and directly visualizes the proximity of the donor (here: Alexa 488-labeled anti-mouse IgG secondary antibody in combination with mouse anti-MIF) and the acceptor molecule (here: Cy3-labelled anti-rabbit secondary antibody in combination with rabbit anti-CXCL4L1). We detected significant donor lifetime shortening (from $2.019 \pm 0.069 \mathrm{~ns}$ to $1.496 \pm 0.033 \mathrm{~ns}$ ) and FRET events (FRET efficiency peak at 20-25\%), when Alexa 488/Cy3 FLIM-FRET was recorded in appropriate regions-of-interest (ROIs) (Figure 5C-D), an observation that is consistent with the notion that MIF and CXCL4L1 not only colocalize in activated platelet preparations but form true heterocomplexes.

To further investigate the physiological relevance of these findings, we next examined clinical thrombus specimens derived from vascular surgery procedures. To determine whether colocalized MIF and CXCL4L1 formed heterocomplexes in thrombus tissue, a proximity ligation assay (PLA) was performed which detects inter-molecular interactions within a distance of $<10 \mathrm{~nm}$. Specific PLA signals were detected in an atherosclerotic thrombus specimen (Figure $6 A-B$ ), suggesting the abundant occurrence of MIF/CXCL4L1 heterocomplexes in the context of clinical thrombus tissue and confirming the FLIM-FRET data obtained in platelet preparations from healthy blood samples. Thus, both FLIM-FRET and PLA demonstrated that MIF and CXCL4L1 form heteromeric complexes upon release from activated platelets. 
1 Heterocomplex formation inhibits MIF-stimulated thrombus formation and alters the 2 effect of MIF on platelet morphology

3 Thrombus formation and clot retraction are relevant processes upon vessel injury and in 4 advanced atherosclerotic vessels. MIF was found to modulate these processes (Wirtz et al, 5 2015). As our data showed that MIF/CXCL4L1 heterocomplexes form in the micro-

6 environment of a thrombus, we next determined whether heterocomplex formation affects 7 thrombus characteristics. Thrombus formation under flow perfusing diluted human blood over 8 a collagen-coated surface harboring combinations of MIF and CXCL4L1 was studied as established (Chatterjee et al, 2014) and was found to double following exposure to MIF when applying a shear rate of $1000 \mathrm{~s}^{-1}$ (Figure 7). CXCL4L1 alone did not affect thrombus characteristics, but when added together with MIF following preincubation, MIF-elicited thrombus formation was blocked. These effects were mainly related to thrombus size/coverage (Figure 7B, Supplementary Figure 5) rather than thrombus numbers (Figure 7C). These data indicated that heterocomplex formation inhibited MIF-stimulated thrombus formation.

The role of platelet morphology and lamellipodia in stable thrombus formation has been controversial, but platelet lamellipodia formation is critical for thrombus formation under flow (Fotinos et al, 2015; Kraemer et al, 2011a; Schurr et al, 2019). To further study the above observed effect of heterocomplex formation on thrombus behavior, we examined the morphology of flow-stressed adhered platelets exposed to MIF or heterocomplexes in detail. Platelet flow stress responses were recorded after 30 and $60 \mathrm{~min}$, with significant changes observed for the 30 min time point. Morphological changes encompassed increased platelet numbers with filopodia, small lamellipodia, large lamellipodia, as well as fully spread platelets. Interestingly, the strong increase in large lamellipodia under control buffer conditions was significantly reduced by MIF and a further significant reduction was observed 26 for platelets coincubated with MIF and CXCL4L1. Inversely, the incubation with the 27 heterocomplex resulted in a significant increase in platelets with small lamellipodia compared 28 to stimulation with MIF alone (Figure $7 D$ ). Figure $7 E$ further illustrates the inverse effect of 
1 MIF/CXCL4L1 on large versus small lamellipodia formation. Together, these experiments 2 indicated MIF/CXCL4L1 heterocomplex formation skewed the morphology of adhering flow3 stressed platelets from a large to a small lamellipodia phenotype compared to treatment with 4 MIF alone.

5

6 
3 Chemokines control numerous pathogenic pathways contributing to inflammation and

\section{Discussion}

atherogenesis. The recent systematic characterization of the chemokine interactome revealed that heteromeric interactions between classical CC- and/or CXC-type chemokines represent an important molecular adjustment screw that serves to amplify, inhibit, or modulate chemokine activity (von Hundelshausen et al., 2017). Here, we have identified a heteromeric interaction between MIF, a pleiotropic inflammatory cytokine and ACK, and the classical platelet chemokine CXCL4L1. We also show that CXCL4L1/MIF complex formation affects inflammatory/atherogenic and thrombogenic activities of MIF. The scheme in Figure 8 summarizes the main findings of this study. This suggests that disease-relevant activities of MIF may be fine-tuned by heterocomplexation with CXCL4L1 and that the chemokine interactome extends to heteromeric interactions between classical and atypical chemokines. In fact, binding of classical chemokines to non-CC- or CXC-chemokine mediators is not unprecendented. Three examples have been documented: i) the CXC-chemokine CXCL12 binds to the alarmin HMGB1 and HMGB1/CXCL12 complex formation promotes chemotactic activity through CXCR4 (De Leo et al., 2019; Schiraldi et al., 2012); ii) the antimicrobial peptide and $\alpha$-defensin HNP1 binds to CCL5 and enhances monocyte adhesion through CCR5 (Alard et al, 2015); iii) macrophage-expressed galectins such as galectin-3 (Gal-3) bind to CXCL12 and attenuate CXCL12-stimulated signaling via CXCR4 (Eckardt et al., 2020). However, while these studies underscore that classical chemokine activity may be modulated by interaction with various soluble mediators, HMGB1 and Gal-3 have no chemotactic activity on their own; HNP1 has been reported to exhibit chemoattractive properties, but the mediating chemoattractant receptor has remained elusive. In contrast, despite lacking the signature structural elements of classical chemokines such as the chemokine-fold and the N-terminal cysteine motif, MIF is a chemoattractant and depending on the microenvironmental context, can signal through the CXC chemokine receptors CXCR2, CXCR4, and/or ACKR3 to promote atherogenic and inflammatory leukocyte recruitment. Its CXC receptor binding capacity is based on the presence of a pseudo-ELR 
1 motif and an extended $\mathrm{N}$-like loop, structurally mimicking the site 1 and 2 receptor binding motifs of the corresponding cognate ligands CXCL1/8 and CXCL12, respectively. Together with the $\beta$-defensins HDB1/2 and HBD3, which bind to CCR6 and CXCR4, respectively, and secreted fragments of certain AARSs, which bind to CXCR1 and CXCR2, MIF has therefore been designated an ACK (Degryse \& de Virgilio, 2003; Kapurniotu et al., 2019; Oppenheim \& Yang, 2005; Rohrl et al., 2010; Sinitski et al., 2019; Wakasugi \& Schimmel, 1999). Our current identification of MIF/CXCL4L1 heterocomplexes thus also shows that the chemokine interactome is not strictly limited to interactions between classical CC- and/or CXC-type chemokines, but also encompasses heteromeric interactions between classical and atypical chemokines, with potential functional modulation of the chemokine receptor pathway of both the classical or atypical chemokine. Although not further validated and pursued in our current study, the detection of additional candidate interactors of MIF in our performed unbiased chemokine array, i.e. CCL28, CXCL9, as well as Prx6 leads us to hypothesize that interactions between classical and atypical chemokines could represent a broader principal of an "expanded ACK/CK interactome".

The validity of the solid phase chemokine array as an unbiased screening approach for candidate chemokine interactors has been previously established (von Hundelshausen et al., 2017). The general utility and specificity of this methodology was further confirmed in the current study. Out of 47 immobilized classical chemokines, in addition to CXCL4L1, only two other classical chemokines, i.e. CCL28 and CXCL9, were revealed to have positivity. While a functional link between MIF and CCL28 has yet to be unveiled, it is interesting to note that the other detected CXC chemokine was CXCL9, a CXCR3 agonist like CXCL4L1. Intriguingly, biotin-MIF neither bound to CXCL12 nor to CXCL8, indicating that implicated functional interactions between MIF and the cognate CXCR4 and CXCR2 ligands, respectively, are independent of heterocomplex formation.

Futhermore, the specificity of the performed array is underscored by the notion that CXCL4, the highly homologous sister variant of CXCL4L1, did not bind to MIF, both at $\mathrm{pH} 8$ and also when we tested for this interaction at $\mathrm{pH} 6$ (data not shown) to account for $\mathrm{pH}$ - 
1 dependent charge differences. We hypothesize that the striking difference between

2 CXCL4L1 and CXCL4 in binding to MIF might be due to the suggested different conformation

3 of these two chemokines, e.g. the more exposed and flexible $\alpha$-helix of monomeric CXCL4L1

4 (Kuo et al., 2013). While CXCL4 has been amply characterized by us and others as a pro-

5 atherogenic platelet chemokine, in part also via its intriguing capacity to hetero-oligomerize

6 with CCL5 (Koenen et al., 2009; von Hundelshausen et al., 2007), very little is known about

7 the role of CXCL4L1 in chronic inflammatory diseases and atherosclerosis. Like its sister

8 molecule, CXCL4L1 is also abundantly expressed in platelets; however, it apparently is not

9 localized in $\alpha$-granules but resides in a different sub-cellular compartment, from where it is 10 constitutively secreted (Lasagni et al, 2007). It is also found in other cell types including 11 mononuclear cells and smooth muscle cells (Lasagni et al., 2007). CXCL4L1 serves as an 12 inhibitor of angiogenesis and has pro-inflammatory effects by inducing the release of CCL2 and CXCL8 from monocytes, while - contrary to CXCL4 - it does not promote monocyte 14 survival (Domschke \& Gleissner, 2019; Gouwy et al, 2016; Sarabi et al, 2011). There is only one in vivo study, in which CXCL4L1 was investigated as prognostic marker in cardiovascular disease. Interestingly, below-median levels of CXCL4L1 were found to correlate with a worse outcome in stable coronary artery disease patients, as indicated by a higher rate of cardiac death, stroke, or myocardial infarction (De Sutter et al, 2012). This finding might argue for a beneficial role of this chemokine in cardiovascular disease, even though the mechanisms behind this remain unclear, but certainly more studies are required. Of note, there is no equivalent of CXCL4L1 in mice (Eisman et al, 1990), limiting functional in vivo studies of this chemokine and its complex with MIF, as predicted from our study. genous pulldown from monocytes, as well as two different biophysical in vitro methods, i.e. SPR and MST. The combination of both methods also addresses potential disadvantages of having one interaction partner immobilized (Zhou et al, 2016). The binding affinity constants derived from the SPR and MST experiments (116 and $160 \mathrm{nM}$, respectively) are in reasonable agreement with each other. The observed (small) difference could be due to a 
1 number of factors, including surface immobilization effects, fluorescence versus biotin

2 labeling, or buffers employed. Together, the results are suggestive of a relatively high binding

3 affinity between MIF and CXCL4L1. Moreover, the obtained nanomolar $K_{D}$ is consistent with

4 the reported concentrations of both proteins in inflammatory disease settings (Sinitski et al.,

5 2019). Flanking evidence for MIF/CXCL4L1 complex formation was obtained by our peptide

6 array mapping and molecular docking results. As expected given their high sequence

7 identity, the peptide array predicted identical binding sites for CXCL4 and CXCL4L1. Also,

8 the peptide array methodology interrogates linear binding epitopes but cannot delineate conformational differences. In fact, Kuo et al. suggested that the three-amino acid difference between CXCL4 and CXCL4L1, although marginal, leads to a slight tilting of the C-terminal $\alpha$-helix (Kuo et al., 2013). We hypothesize that this moderate conformational change could be the basis for the observed preferred binding of MIF to CXCL4L1 compared to CXCL4. Differences in their binding affinity to CCL5 have already been reported for CXCL4 and CXCL4L1 and also the availability of their monomers, regulated by the stability of their tetrameric complexes, differs between these two chemokines (Sarabi et al., 2011). Future structural studies, e.g. by nuclear magnetic resonance (NMR) spectroscopy, may help to further address these and other conformational questions.

To investigate the functional consequences of MIF/CXCL4L1 heterocomplex formation, we focused on inflammatory and atherosclerosis-relevant activities of MIF. T-cell migration is one such activity that is regulated by the MIF/CXCR4 pathway (Bernhagen et al., 2007). In line with previous results, MIF promoted T-cell migration in a physiologically relevant $3 \mathrm{D}$ migration setting. Although $\mathrm{T}$ cells generally express the CXCL4L1 receptor CXCR3, CXCL4L1 alone had no effect on the chemotaxis of human PBMC-derived T cells. Lack of CXCL4L1 activity in this assay is likely due to the fact that CXCL4L1 is not a bona fide T-cell chemoattractant (Gouwy et al., 2016) and that the preferential CXCL4L1 receptor variant CXCR3B is poorly expressed on T cells (Korniejewska et al, 2011). The 3D T-cell migration data are supported by the result that MIF, but not the combination of MIF and CXCL4L1, promoted Jurkat T-cell migration in a 2D Transwell assay. Confirming the 
remarkable specificity of MIF binding to CXCL4L1 versus CXCL4, coincubation of MIF with CXCL4 did not result in reduced Jurkat T-cell migration. Of note, heterocomplex formation with MIF led to a complete blockade of MIF's pro-migratory effect on primary T cells in the 3D migration setting. While in vivo T-cell recruitment studies were beyond the scope of our study, inhibition of MIF-mediated T-cell migration by CXCL4L1 complexation could potentially be relevant in atherosclerosis, where it might represent a feedback mechanism that could serve to dampen the atherogenic response. In fact, abundant CXCL4L1 levels may be released by activated platelets in an atherogenic microenvironment, where they could colocalize with endothelial-immobilized or monocyte-secreted MIF and infiltrating T cells. That complexation of MIF by CXCL4L1 can interfere with MIF's chemoattractant activities was confirmed in a microglia assay, in which the motility of Egfp ${ }^{+}$microglia in murine cortical brain cultures ex vivo was studied. In addition to representing an independent cell migration system, the data obtained from the microglia-containing cortical cultures further confirmed that complex formation interferes with MIF signaling through the CXCR4 pathway and underscored that the mechanism could be relevant in in vivo-like physiological tissue settings. That MIF/CXCL4L1 heterocomplex formation interferes with MIF signaling through CXCR4 was independently validated by biochemical experiments using FP spectroscopy and DMR analysis of HEK293-CXCR4 transfectants.

This identified interaction of MIF with CXCL4L1, supposedly resulting in local inhibition of MIF's pro-inflammatory effects was especially interesting to us in the context of previous studies, in which we identified human and mouse platelets as an abundant source of MIF (Strüßmann et al., 2013; Wirtz et al., 2015). Here, we verified expression and localization of MIF in human platelets as well as in platelet-rich clinical thrombus tissue by confocal (CLSM) and multiphoton microscopy (MPM). As expected, these experiments also showed the abundant presence of CXCL4L1 in platelets and thrombi, and suggested the colocalization and/or complex formation of MIF and CXCL4L1 in the vicinity of platelets. Due to the optical resolution limits of the CLSM and MPM methods, true colocalization and the specific subcellular compartment could not be determined. Evidence for the presence of 
1 MIF/CXCL4L1 heteromers is suggested by PLA performed on cryosections of a human 2 thrombus. In fact, PLA is an established method to detect CK heteromers as shown 3 previously for HNP1/CCL5 complexes (Alard et al., 2015).

Having confirmed the occurrence of this novel complex in platelet preparations and 5 thrombus tissue, lastly the effect of MIF, CXCL4L1 and their complex on platelet function and 6 thrombus formation was assessed. MIF promoted thrombus formation leading to a larger 7 thrombus-covered area in an in vitro setting under flow conditions. Confirming our previous results, this effect was abrogated upon co-incubation with CXCL4L1. It is interesting to note that in the settings used in our experiment applying a shear rate of $1000 \mathrm{~s}^{-1}$ for $5 \mathrm{~min}$, MIF acted to enhance thrombus formation. Instead, in a previous study employing a shear rate of $1700 \mathrm{~s}^{-1}$ MIF was found to reduce thrombus size, confirming that MIF is a modulator of thrombus formation, but also indicating that the directionality of the effect may depend on the specific microenvironmental context. activation on a fibrinogen-coated surface revealed that both MIF and CXCL4L1 favored a switch from large to small lamellipodia at an early time point. Interestingly, in this setting no inhibition by the complex on MIF-mediated effects was observed, but a synergistic behavior of MIF and CXCL4L1 was observed, suggesting that this effect may occur independently of CXCR4.

In addition to their classical role in wound closure and haemostasis, thrombus formation and platelet activation are processes that are closely linked to inflammatory processes driving atherosclerosis (Gawaz, 2006; Lippi et al, 2011; Nording et al, 2020; von Hundelshausen \& Weber, 2007). MIF has been amply linked to atherosclerotic pathogenesis both clinically and experimentally, with evidence for a number of contributing mechanisms including leukocyte recruitment and platelet activation (Bernhagen et al., 2007; Chatterjee et al., 2014; Muller et al, 2013; Sinitski et al., 2019; Zernecke et al, 2008) The identified 27 heteromerization of MIF and CXCL4L1 in our current study and the observed effect of MIF/CXCL4L1 complex formation on immune cell migration as well as thrombus size and 
1 platelet morphology might imply that CXCL4L1 could have a protective role in atherosclerosis

2 by mitigating the pro-atherosclerotic effects of MIF via complex formation. This hypothesis

3 would warrant future studies in corresponding experimental in vivo models, albeit the lack of

$4 \quad$ CXCL4L1 expression in rodents will impose a particular challenge here.

In summary, we provide evidence that MIF does not only behave as a chemokine-like

6 mediator by way of engaging classical chemokine receptors but also by direct binding to

7 classical chemokines. Interestingly, the identified chemokine interactor of MIF is not one of

8 the cognate ligands of the MIF receptors CXCR2 or CXCR4, but CXCL4L1, a prominent

9 platelet chemokine not previously implicated in MIF biology or MIF-mediated pathologies.

10 While evidence from experimental in vivo disease models will have to be obtained in future 11 studies, our data suggest that MIF/CXCL4L1 complex formation could serve to attenuate inflammatory/atherogenic activities of MIF through the CXCR4 receptor axis. Our study also

13 gives insight into the growing "chemokine interactome" with a particular focus on ACKs.

14 While modulatory effects on the interactome by mediators not belonging to the class of chemokines have already been exemplified by intriguing studies involving HMGB1, HNP1, and the galectins (Alard et al., 2015; Eckardt et al., 2020; Schiraldi et al., 2012), the current study is first in demonstrating a role for MIF family proteins in particular, and bona fide ACKs in general, as defined by their chemotactic activity mediated through engagement of classical chemokine receptors. While not yet validated by follow up analyses, the identification of additional potential interactors in our array indicates that this could represent a broader 21 principle of an ACK/CK interactome. 


\section{Figure legends}

Figure 1: Unbiased chemokine protein array identifies CXCL4L1, but not CXCL4, as a novel

interaction candidate of MIF. (A) Schematic illustrating binding of biotinylated MIF to the chemokine protein array. (B) Layout of the immobilized chemokines, atypical chemokines and alarmins (top) and membrane of chemokine solid phase assay performed at $\mathrm{pH}$ 8.0, developed against bound biotin-MIF (bottom). (C) Close-up of the membrane with a focus on CXCL4 and CXCL4L1 with the corresponding negative control membrane, incubated without biotin-MIF.

Figure 2: Validation of MIF/CXCL4L1 complex formation by a variety of protein-protein interaction assays and verification of the specificity of MIF complexation with CXCL4L1 over CXCL4. (A) Semi-endogenous pull-down assay, in which endogenous CXCL4L1 from MonoMac6 lysates was captured with recombinant biotinylated MIF and pulled down by streptavidin-coated paramagnetic beads. Blots, developed against MIF (left) and CXCL4L1 (right), show representative results of three independent experiments. Input corresponds to $5 \%$ cell lysate without pull-down and control (Ctrl) refers to pull-downs performed in the absence of biotin-MIF. Molecular weight markers were lelectrophoresed in the same gel and relevant marker sizes are indicated. (B) Interrogation of MIF/CXCL4L1 complex formation by surface plasmon resonance (SPR) spectroscopy using chip-immobilized biotin-MIF titred against increasing concentrations of CXCL4L1. Measurements indicate an interaction between MIF and CXCL4L1 with an estimated $K_{D}$ of $116 \pm 16 \mathrm{nM}$. The SPR response signal is given in relative units $(R U)$. (C) Same as (B), except that titration was performed with CXCL4. Corresponding SPR spectroscopy data for MIF and CXCL4. No detectable binding signal was obtained and no $K_{D}$ could be derived. (D) Interrogation of MIF/CXCL4L1 complex formation by microscale thermophoresis (MST) utilizing fluorescently labeled MIF and CXCL4L1 in solution. MST analysis revealed a $K_{D}$ of $159.8 \pm 16.8 \mathrm{nM}$ for the interaction of 
1 MIF and CXCL4L1. (E) Same as (D), except that CXCL4 was tested. The derived apparent

$2 \mathrm{~K}_{\mathrm{D}}$ of $2.0 \pm 0.8 \mu \mathrm{M}$ was ten-fold higher compared to MIF/CXCL4L1.

4 Figure 3. Co-incubation with CXCL4L1 inhibits MIF-mediated immune cell chemotaxis. (A) Migration of human CD4 ${ }^{+}$T-cells embedded in a gel matrix, subjected to gradients of MIF, CXCL4L1 or both. Movement of cells was followed by live cell imaging and individual tracks reconstructed from acquired images. Tracks of cells migrating towards the indicated stimuli are marked in the corresponding color. Starting point was centered to $x=y=0$. The black crosshair indicates the cell population's center of mass after migration. (B) Quantification of the $3 \mathrm{D}$ chemotaxis experiment in $(\mathbf{A})$, indicating that complexation of MIF by CXCL4L1 attenuates MIF-mediated directed migration of human CD4 ${ }^{+}$T-cells. Plotted is the calculated forward migration index (FMI), based on manual tracking of at least 30 individual cells per treatment. (C) Migration trajectories of murine microglia, obtained by live cell imaging for 15 h, treated with MIF, CXCL4L1, or both. Used concentrations: MIF: 8 nM, CXCL4L1: $1.6 \mathrm{nM}$; $\mathrm{n}=5$ independent experiments; horizontal bar: $100 \mu \mathrm{m}$. (D) Analysis of microglia motility, based on each tracked cell accumulated distance, shown in (C). Data is presented as mean \pm SD. Statistical significance is indicated as described: ${ }^{*}, \mathrm{P}<0.05 ;{ }^{* *}, \mathrm{P}<0.01$; ${ }^{* *}, \mathrm{P}<$ 0.001 .

Figure 4. MIF/CXCL4L1 complex formation inhibits binding of MIF to CXCR4 and signaling of MIF through the CXCR4 signaling axis. (A) Fluorescence polarization (FP) spectroscopy shows the interaction of Alexa488-labeled MIF with the soluble CXCR4 receptor mimic msR4M-L1 with an apparent $K_{D}$ of $237.2 \pm 24.2 \mathrm{nM}$. Data is presented as mean of 3 independent experiments; error bars represent the SD.

(B) Pre-incubation of MIF with CXCL4L1 (160-fold molar excess) prevents the interaction of MIF with msR4M-L1 (app. $\mathrm{K}_{\mathrm{D}}>$ $10 \mu \mathrm{M})$. Mean of 3 experiments \pm SD.

(C) Dynamic mass redistribution (DMR)

27 measurements with HEK293 cells stably expressing CXCR4 indicate that the cellular 
1 of CXCR4-expressing HEK293 cells to MIF in the presence or absence of the CXCR42 antagonist AMD3100 is also shown, confirming the CXCR4-dependency of the cellular 3 response to MIF.

4

Figure 5. Co-localization and interaction of MIF and CXCL4L1 in human platelet preparations, detected in multiphoton microscopy (MPM). (A): MPM images of isolated platelets, forming small aggregates, stained for MIF and CXCL4L1. White arrowheads indicate areas of colocalization. Size bar: $5 \mu \mathrm{m}$. (B) MPM images of isolated, more separated platelets, stained as in (A), showing colocalization of MIF and CXCL4L1. Size bar: $5 \mu \mathrm{m}$. (C) Fluorescence lifetime imaging (FLIM) of platelets isolation as shown in (B). Color-code corresponds to lifetime of the donor, Alexa 488, the dye used for the antibody-based staining of MIF. (D) Histogram of the Förster Resonance Energy Transfer (FRET) efficiency in (C). (E) Donor lifetime shortening, presented as the mean lifetime ( $\mathrm{T}$ ), average weighted, of the donor (Alexa 488, MIF staining) alone, and in combination with the acceptor fluorophore (Cy3, CXCL4L1 staining), where FRET occured.

Figure 6. Proximity ligation assay (PLA) indicates that MIF/CXCL4L1 heterocomplexes are present in human thrombus tissue. (A) MIF/CXCL4L1 complex formation in thrombus specimen revealed by PLA. PLA-positive signals are depicted in yellow; tissue was counterstained with fluorescent-labeled phalloidin (cyan). Stained tissue samples were imaged by CLSM; size bar: $50 \mu \mathrm{m}$. (B) HE staining of thrombus tissue specimen; size bar: 75 $\mu \mathrm{m}$.

Figure 7. (A) Thrombus formation in human blood under flow stress is enhanced by MIF, and this effect is diminished by pre-incubation of MIF with CXCL4L1. Fluorescent staining with $\mathrm{DiOC}_{6}$. Shown are representative images of one experiment, performed at a shear rate of $1000 \mathrm{~s}^{-1}$; size bar: $100 \mu \mathrm{m}$. (B) Quantification of thrombi sizes from flow chamber experiments, as depicted exemplarily in (A). MIF-mediated increase in thrombus-covered 
1 area is diminished, when MIF is pre-incubated with CXCL4L1. $n=6$ experiments and

2 platelets coming from 4 donors. (C) Quantification of total thrombi numbers per treatment

3 group. As thrombus numbers remain unchanged, effects on thrombus-covered area originate

4 from the size of the formed thrombi (see also Supplementary Figure 5); $n=6$ experiments.

5 (D) Analysis and quantification of platelet morphology upon adhesion on fibrinogen-coated

6 coverslips. Activated platelets were allowed to adhere on fibrinogen-coated coverslips that

7 were pre-treated with MIF, CXCL4L1 or a mixture of both for the indicated times. After fixing

8 with PFA, images of randomly selected areas were taken and platelet morphology analyzed.

9 Treatment with a combination of MIF and CXCL4L1 led to a reduction in the large 10 lamellopodia phenotype, favoring small lamellopodia, with the MIF/CXCL4L1 complex 11 showing a stronger effect then the individual proteins; $n=6$ experiments. (E) Platelet morphology distribution after 30 min for each treatment group according to panel (D).

14 Figure 8: Summary scheme and suggested model of CXCL4L1/MIF complex formation and 15 functions. The atypical chemokine MIF and the classical chemokine CXCL4L1, e.g. present 16 in an inflammatory or atherogenic microenvironment after release from platelets, form 17 heteromeric complexes. Complexes inhibit inflammatory effects of MIF on leukocyte 18 recruitment as well as its pro-thrombotic effects through impairing MIF interactions with its 19 non-cognate receptor CXCR4. 


\section{Supplementary figure legends}

Supplementary Figure 1. Additional data for chemokine protein array. (A) Negative control membrane related to the experiment in Figure 1, incubated in buffer at $\mathrm{pH} 8.0$ without biotinMIF. (B) Close-up of membrane from a chemokine protein array experiment with a focus on CXCL4 and CXCL4L1. The membrane was incubated with biotin-MIF and the incubation was performed at $\mathrm{pH} 6.0$.

Supplementary Figure 2. Investigation of the MIF/CXCL4L1 interaction interface and in silico studies. (A) CelluSpot peptide array experiments, where overlapping peptides of CXCL4 (left) and CXCL4L1 (right) were spotted on an array and probed with biotin-MIF. Chemiluminescence signal intensity indicates binding of biotin-MIF to the respective peptide. Arrows indicate peptides of interest that are most likely to be involved in the interaction with MIF. (B) Sequences of peptides identified in A are highlighted in the 3D structure of monomeric CXCL4 and CXCL4L1, showing their localization in the folded proteins. For both chemokines, these peptides of interest represent almost identical amino acid sequences, corresponding to highly similar regions of the protein. This indicates that not only the sequence but also the three-dimensional conformation of the chemokines might play a role in the interaction with MIF. Amino acid residues, in which CXCL4L1 differs from CXCL4 are in italics. PyMOL was used to visualize a CXCL4 (PDB ID: 1F9Q Chain A) and CXCL4L1 monomer (PDB ID: 4HSV Chain A). (C) To visualize the proposed MIF/CXCL4L1 complex, an unbiased in silico protein-protein docking approach was taken. The ClusPro 2.0 webserver was used to simulate a complex consisting of both a MIF and CXCL4L1 monomer. Depicted here is the highest-ranking docking result, with peptides identified in $\mathbf{A}$ to be potentially part of the interaction interface highlighted in CXCL4L1. According to this in silico prediction, they are partially directed towards MIF, allowing parts of their sequences being involved in complex formation. PyMOL was used to calculate the surface charge distribution of these proteins (red: negatively charged; blue: positively charged), revealing an 
1 area of opposite charges in the proposed contact region of MIF and CXCL4L1 that partially

2 matches the peptide array results.

3

4 Supplementary Figure 3. Effects on cell migration in Jurkat T cells and microglia. (A) Effect 5 of CXCL4L1 on MIF-mediated chemotaxis of Jurkat T cells as analyzed in a Transwell 6 migration assay. Used concentrations: MIF: $16 \mathrm{nM}, \mathrm{CXCL} 4 \mathrm{~L} 1: 32 \mathrm{nM}$; Data is presented as 7 mean \pm SD. $n=2-4$ independent experiments. (B) Same as $(\mathbf{A})$, except that co-incubation 8 with CXCL4 was analyzed. Data is presented as mean \pm SD. $n=4$ independent experiments 9 with duplicates each. (C) Quantification of murine microglia motility, based on the 10 accumulated distance of GFP-positive microglia tracked during live cell imaging $(n=5)$. MIF 11 was used at a concentration of $8 \mathrm{nM}$, the soluble CXCR4-mimicking peptide msR4M-L1 at 40 $\mathrm{nM}$ and the cognate ligand of CXCR4, CXCL12, at $16 \mathrm{nM}$. Data presented as mean \pm SD.

13 Statistical significance: ${ }^{*}, \mathrm{P}<0.05 ;{ }^{* *}, \mathrm{P}<0.01 ;{ }^{* * *}, \mathrm{P}<0.005 ;{ }^{* * *}, \mathrm{P}<0.0001$.

Supplementary Figure 4. Quantification of mean thrombus sizes from Figure 5A, showing a trend for CXCL4L1 inhibiting the MIF-mediated increase in thrombus size in samples, in which MIF and CXCL4L1 were pre-incubated together. 


\section{Declarations}

2

\section{Ethics approval and consent to participate}

Mouse maintenance and experiments were reviewed and overseen by the institutional animal use and care committee of the local authorities (Regierung von Oberbayern, ROB, Germany) and performed in accordance with the procedures provided by the animal protection representative of CSD. Human thrombus tissue specimens were obtained as disposable material from vascular surgery procedures under ethics allowance LMU Munich 18-104 and TUM-MRI project \# 2799/10).

\section{Consent for publication}

$3-N / A-$

4

$5 \quad$ Availability of data and material

All data and materials as well as software application information are available in the manuscript, the supplementary information, or are available from the corresponding authors upon reasonable request.

Competing interests

J.B., P.v.H., C.W., and A.K. are inventors on patent applications related to anti-MIF and antichemokine strategies in inflammatory and cardiovascular diseases. The other authors declare that they have no competing interests.

\section{Funding}

16 This work was supported by Deutsche Forschungsgemeinschaft (DFG) grant SFB1123-A3 to 17 J.B. and A.K., DFG INST 409/209-1 FUGG to J.B., SFB1123-A2 to P.v.H., SFB1123-A1 to 18 C.W., SFB1123-B5 to L.M., SFB240-B01 (374031971 - TRR 240) to M.G., and by DFG 19 under Germany's Excellence Strategy within the framework of the Munich Cluster for 
1 Systems Neurology (EXC 2145 SyNergy_ID 390857198) to J.B., C.W., and O.G. A.H. was

2 supported by a Metiphys scholarship of LMU Munich and by the Friedrich-Baur-Foundation

3 e.V. at LMU University Hospital. E.S. and J.B. received funding from the LMU-FöFoLe 4 program under project 52114016.

\section{Authors' contributions}

7 Jürgen Bernhagen, Philipp von Hundelshausen, Markus, Brandhofer, Adrian Hoffmann, and 8 Xavier Blanchet conceived and designed the study with help from Christian Weber, Aphrodite 9 Kapurniotu, Meinrad Gawaz, Ozgun Gokce, Remco T.A. Megens, Hans Ippel, Rory R. Koenen, and Kevin Mayo. Markus Brandhofer, Adrian Hoffmann, Xavier Blanchet, Elena Siminkovitch, Anne-Katrin Rohlfing, Omar El Bounkari, Jeremy A. Nestele, Alexander Bild, Christos Kontos, Kathleen Hille, Vanessa Rohde, Adrian Fröhlich, Jona Golemi, and Christine Krammer performed research and analyzed data. Markus Brandhofer, Adrian Hoffmann, Xavier Blanchet, Elena Siminkovitch, Anne-Katrin Rohlfing, Christian Weber, Meinrad Gawaz, Aphrodite Kapurniotu, Omar El Bounkari, Philipp von Hundelshausen, and Jürgen Bernhagen contributed to the interpretation of the data. The first draft of the manuscript was written by Markus Brandhofer and Jürgen Bernhagen with help from Adrian Hoffmann and Philipp von Hundelshausen. All authors revised and commented on the manuscript drafts. Patrick Scheiermann, Wolfgang E. Kempf, Lars Maegdefessel, and Rory Koenen contributed to critical materials, and Jürgen Bernhagen, Philipp von Hundelshausen, Christian Weber, Aphrodite Kapurniotu, Remco T.A. Megens, Adrian Hoffmann, Meinrad Gawaz, and Lars Maegdefessel provided funding for the study.

\section{Acknowledgements}

C.W. is Van de Laar Professor of Atherosclerosis. We thank Simona Gerra, Priscila Bourilhon, and Lusine Saroyan for technical support. We are grateful to the mouse core facility of the Center for Stroke and Dementia Research (CSD) for their support. We also 
1 thank the Biophysics Core Facility at the School of Biology of LMU Munich and Dr. Sophie 2 Brameyer for usage of the MST instrument.

3

4 Authors' information

$5 \quad \mathrm{~N} / \mathrm{A}$

6 


\section{References}

Alard JE, Ortega-Gomez A, Wichapong K, Bongiovanni D, Horckmans M, Megens RT, Leoni G, Ferraro B, Rossaint J, Paulin N et al (2015) Recruitment of classical monocytes can be inhibited by disturbing heteromers of neutrophil HNP1 and platelet CCL5. Sci Trans/ Med 7: 317 ra196

Bachelerie F, Ben-Baruch A, Burkhardt AM, Combadiere C, Farber JM, Graham GJ, Horuk R, Sparre-Ulrich AH, Locati M, Luster AD et al (2014a) International Union of Basic and Clinical Pharmacology. LXXXIX. Update on the extended family of chemokine receptors and introducing a new nomenclature for atypical chemokine receptors. Pharmacol Rev 66: 1-79

Bachelerie F, Graham GJ, Locati M, Mantovani A, Murphy PM, Nibbs R, Rot A, Sozzani S, Thelen M (2014b) New nomenclature for atypical chemokine receptors. Nat Immunol 15: 207-208

Bai F, Asojo OA, Cirillo P, Ciustea M, Ledizet M, Aristoff PA, Leng L, Koski RA, Powell TJ, Bucala $\mathrm{R}$ et al (2012) A novel allosteric inhibitor of macrophage migration inhibitory factor (MIF). J Biol Chem 287: 30653-30663

Bernhagen J, Krohn R, Lue H, Gregory JL, Zernecke A, Koenen RR, Dewor M, Georgiev I, Schober A, Leng L et al (2007) MIF is a noncognate ligand of CXC chemokine receptors in inflammatory and atherogenic cell recruitment. Nat Med 13: 587-596

Bernhagen J, Mitchell RA, Calandra T, Voelter W, Cerami A, Bucala R (1994) Purification, bioactivity, and secondary structure analysis of mouse and human macrophage migration Inhibitory factor (MIF). Biochemistry 33: 14144-14155

Borst O, Schmidt EM, Munzer P, Schonberger T, Towhid ST, Elvers M, Leibrock C, Schmid E, Eylenstein A, Kuhl D et al (2012) The serum- and glucocorticoid-inducible kinase 1 (SGK1) influences platelet calcium signaling and function by regulation of Orai1 expression in megakaryocytes. Blood 119: 251-261 
1 Calandra T, Roger T (2003) Macrophage migration inhibitory factor: a regulator of innate

2 immunity. Nat Rev Immunol 3: 791-800

3 Charo IF, Ransohoff RM (2006) The many roles of chemokines and chemokine receptors in 4 inflammation. N Engl J Med 354: 610-621

5 Chatterjee M, Borst O, Walker B, Fotinos A, Vogel S, Seizer P, Mack A, Alampour-Rajabi S, 6 Rath D, Geisler T et al (2014) Macrophage migration inhibitory factor limits activation7 induced apoptosis of platelets via CXCR7-dependent Akt signaling. Circ Res 115: 939-949

8 David JR (1966) Delayed hypersensitivity in vitro: its mediation by cell-free substances 9 formed by lymphoid cell-antigen interaction. Proc Natl Acad Sci U S A 56: 72-77

10 De Leo F, Quilici G, Tirone M, De Marchis F, Mannella V, Zucchelli C, Preti A, Gori A, 11 Casalgrandi M, Mezzapelle $\mathrm{R}$ et al (2019) Diflunisal targets the HMGB1/CXCL12 heterocomplex and blocks immune cell recruitment. EMBO Rep 20: e47788

De Sutter J, Van de Veire NR, Struyf S, Philippe J, De Buyzere M, Van Damme J (2012) PF4var/CXCL4L1 predicts outcome in stable coronary artery disease patients with preserved left ventricular function. PLoS One 7: e31343

Degryse B, de Virgilio M (2003) The nuclear protein HMGB1, a new kind of chemokine? FEBS Lett 553: 11-17

Domschke G, Gleissner CA (2019) CXCL4-induced macrophages in human atherosclerosis. Cytokine 122: 154141

Eckardt V, Miller MC, Blanchet X, Duan R, Leberzammer J, Duchene J, Soehnlein O, Megens RT, Ludwig AK, Dregni A et al (2020) Chemokines and galectins form heterodimers to modulate inflammation. EMBO Rep 21: e47852

Eiger DS, Boldizsar N, Honeycutt CC, Gardner J, Rajagopal S (2021) Biased agonism at chemokine receptors. Cell Signal 78: 109862 
1 Eisman R, Surrey S, Ramachandran B, Schwartz E, Poncz M (1990) Structural and

2 functional comparison of the genes for human platelet factor 4 and PF4alt. Blood 76: 336$3 \quad 344$

4 Fotinos A, Klier M, Gowert NS, Munzer P, Klatt C, Beck S, Borst O, Billuart P, Schaller M,

5 Lang $\mathrm{F}$ et al (2015) Loss of oligophrenin1 leads to uncontrolled Rho activation and increased

6 thrombus formation in mice. J Thromb Haemost 13: 619-630

7 Gawaz M (2006) Platelets in the onset of atherosclerosis. Blood Cells Mol Dis 36: 206-210

8 Gokce O, Sudhof TC (2013) Membrane-tethered monomeric neurexin LNS-domain triggers

9 synapse formation. J Neurosci 33: 14617-14628

Gouwy M, Ruytinx P, Radice E, Claudi F, Van Raemdonck K, Bonecchi R, Locati M, Struyf S (2016) CXCL4 and CXCL4L1 Differentially affect monocyte survival and dendritic cell differentiation and phagocytosis. PLoS One 11: e0166006

Hutchings CJ, Koglin M, Olson WC, Marshall FH (2017) Opportunities for therapeutic antibodies directed at G-protein-coupled receptors. Nat Rev Drug Discov 16: 787-810

Kapurniotu A, Gokce O, Bernhagen J (2019) The multitasking potential of alarmins and atypical chemokines. Front Med (Lausanne) 6: 3

Karshovska E, Weber C, von Hundelshausen P (2013) Platelet chemokines in health and disease. Thromb Haemost 110: 894-902

Klasen C, Ohl K, Sternkopf M, Shachar I, Schmitz C, Heussen N, Hobeika E, Levit-Zerdoun E, Tenbrock K, Reth M et al (2014) MIF promotes B cell chemotaxis through the receptors CXCR4 and CD74 and ZAP-70 signaling. J Immunol 192: 5273-5284

Kleist AB, Getschman AE, Ziarek JJ, Nevins AM, Gauthier PA, Chevigne A, Szpakowska M, Volkman BF (2016) New paradigms in chemokine receptor signal transduction: Moving beyond the two-site model. Biochem Pharmacol 114: 53-68 
1 Koenen RR, von Hundelshausen P, Nesmelova IV, Zernecke A, Liehn EA, Sarabi A, Kramp

2 BK, Piccinini AM, Paludan SR, Kowalska MA et al (2009) Disrupting functional interactions

3 between platelet chemokines inhibits atherosclerosis in hyperlipidemic mice. Nat Med 15: 97-

$4 \quad 103$

5 Kontos C, El Bounkari O, Krammer C, Sinitski D, Hille K, Zan C, Yan G, Wang S, Gao Y,

6 Brandhofer M et al (2020) Designed CXCR4 mimic acts as a soluble chemokine receptor that

7 blocks atherogenic inflammation by agonist-specific targeting. Nat Commun 11: 5981

8 Korniejewska A, McKnight AJ, Johnson Z, Watson ML, Ward SG (2011) Expression and 9 agonist responsiveness of CXCR3 variants in human T lymphocytes. Immunology 132: 503515

Kozakov D, Hall DR, Xia B, Porter KA, Padhorny D, Yueh C, Beglov D, Vajda S (2017) The ClusPro web server for protein-protein docking. Nat Protoc 12: 255-278

Kraemer BF, Schmidt C, Urban B, Bigalke B, Schwanitz L, Koch M, Seizer P, Schaller M, Gawaz M, Lindemann S (2011a) High shear flow induces migration of adherent human platelets. Platelets 22: 415-421

Kraemer S, Lue H, Zernecke A, Kapurniotu A, Andreetto E, Frank R, Lennartz B, Weber C, Bernhagen J (2011b) MIF-chemokine receptor interactions in atherogenesis are dependent on an N-loop-based 2-site binding mechanism. FASEB J 25: 894-906

Krammer C, Kontos C, Dewor M, Hille K, Dalla Volta B, El Bounkari O, Tas K, Sinitski D, Brandhofer M, Megens RTA et al (2021) A MIF-Derived Cyclopeptide that Inhibits MIF Binding and Atherogenic Signaling via the Chemokine Receptor CXCR2. Chembiochem 22: $1012-1019$

Kuo JH, Chen YP, Liu JS, Dubrac A, Quemener C, Prats H, Bikfalvi A, Wu WG, Sue SC (2013) Alternative C-terminal helix orientation alters chemokine function: structure of the antiangiogenic chemokine, CXCL4L1. J Biol Chem 288: 13522-13533 
1 Lacy M, Kontos C, Brandhofer M, Hille K, Groning S, Sinitski D, Bourilhon P, Rosenberg E,

2 Krammer C, Thavayogarajah $\mathrm{T}$ et al (2018) Identification of an Arg-Leu-Arg tripeptide that

3 contributes to the binding interface between the cytokine MIF and the chemokine receptor

$4 \quad$ CXCR4. Sci Rep 8: 5171

5 Lasagni L, Grepin R, Mazzinghi B, Lazzeri E, Meini C, Sagrinati C, Liotta F, Frosali F,

6 Ronconi E, Alain-Courtois $\mathrm{N}$ et al (2007) PF-4/CXCL4 and CXCL4L1 exhibit distinct

7 subcellular localization and a differentially regulated mechanism of secretion. Blood 109:

$8 \quad 4127-4134$

9 Leng L, Metz CN, Fang Y, Xu J, Donnelly S, Baugh J, Delohery T, Chen Y, Mitchell RA,

10 Bucala R (2003) MIF signal transduction initiated by binding to CD74. J Exp Med 197: 1467-

$11 \quad 1476$

Lippi G, Franchini M, Targher G (2011) Arterial thrombus formation in cardiovascular disease. Nat Rev Cardiol 8: 502-512

Ma F, Kouzoukas DE, Meyer-Siegler KL, Hunt DE, Leng L, Bucala R, Vera PL (2017) Macrophage migration inhibitory factor mediates protease-activated receptor 4-induced bladder pain through urothelial high mobility group box 1. Physiol Rep 5: e13549.

Michelet C, Danchin EGJ, Jaouannet M, Bernhagen J, Panstruga R, Kogel KH, Keller H, Coustau C (2019) Cross-Kingdom Analysis of Diversity, Evolutionary History, and Site Selection within the Eukaryotic Macrophage Migration Inhibitory Factor Superfamily. Genes (Basel) 10: 740

Moerke NJ (2009) Fluorescence Polarization (FP) Assays for Monitoring Peptide-Protein or Nucleic Acid-Protein Binding. Curr Protoc Chem Biol 1: 1-15

Muller I, Schonberger T, Schneider M, Borst O, Ziegler M, Seizer P, Leder C, Muller K, Lang M, Appenzeller F et al (2013) Gremlin-1 Is an inhibitor of macrophage migration inhibitory 
1 factor and attenuates atherosclerotic plaque growth in ApoE-/- Mice. J Biol Chem 288:

$2 \quad 31635-31645$

3 Murphy PM, Baggiolini M, Charo IF, Hebert CA, Horuk R, Matsushima K, Miller LH,

4 Oppenheim JJ, Power CA (2000) International union of pharmacology. XXII. Nomenclature

5 for chemokine receptors. Pharmacol Rev 52: 145-176

6 Nibbs RJ, Graham GJ (2013) Immune regulation by atypical chemokine receptors. Nat Rev 7 Immunol 13: 815-829

8 Niess JH, Brand S, Gu X, Landsman L, Jung S, McCormick BA, Vyas JM, Boes M, Ploegh

9 HL, Fox JG et al (2005) CX3CR1-mediated dendritic cell access to the intestinal lumen and 10 bacterial clearance. Science $307: 254-258$

11 Noels H, Weber C, Koenen RR (2019) Chemokines as therapeutic targets in cardiovascular disease. Arterioscler Thromb Vasc Biol 39: 583-592

13 Nording H, Baron L, Langer HF (2020) Platelets as therapeutic targets to prevent 14 atherosclerosis. Atherosclerosis 307: 97-108

15 Oppenheim JJ, Yang D (2005) Alarmins: chemotactic activators of immune responses. Curr Opin Immunol 17: 359-365

17 Pawig L, Klasen C, Weber C, Bernhagen J, Noels H (2015) Diversity and Inter-Connections 18 in the CXCR4 Chemokine Receptor/Ligand Family: Molecular Perspectives. Front Immunol $196: 429$ Polack B, Schved JF, Boneu B, Groupe d'Etude sur l'Hemostase et la T (2001) Preanalytical recommendations of the 'Groupe d'Etude sur l'Hemostase et la Thrombose' (GEHT) for venous blood testing in hemostasis laboratories. Haemostasis 31: 61-68 
1 Interactions: evidence for partial allosteric agonism in comparison with CXCL12 chemokine.

2 J Biol Chem 291: 15881-15895

3 Rohrl J, Yang D, Oppenheim JJ, Hehlgans T (2010) Specific binding and chemotactic activity

4 of mBD4 and its functional orthologue hBD2 to CCR6-expressing cells. J Biol Chem 285:

$5 \quad 7028-7034$

6 Sarabi A, Kramp BK, Drechsler M, Hackeng TM, Soehnlein O, Weber C, Koenen RR, Von

7 Hundelshausen P (2011) CXCL4L1 inhibits angiogenesis and induces undirected endothelial

8 cell migration without affecting endothelial cell proliferation and monocyte recruitment. $J$

$9 \quad$ Thromb Haemost 9: 209-219

10 Schiraldi M, Raucci A, Munoz LM, Livoti E, Celona B, Venereau E, Apuzzo T, De Marchis F, 11 Pedotti M, Bachi A et al (2012) HMGB1 promotes recruitment of inflammatory cells to 12 damaged tissues by forming a complex with CXCL12 and signaling via CXCR4. J Exp Med 13 209: $551-563$

14 Schroder R, Janssen N, Schmidt J, Kebig A, Merten N, Hennen S, Muller A, Blattermann S, 15 Mohr-Andra M, Zahn S et al (2010) Deconvolution of complex G protein-coupled receptor 16 signaling in live cells using dynamic mass redistribution measurements. Nat Biotechnol 28: $17 \quad 943-949$

Schurr Y, Sperr A, Volz J, Beck S, Reil L, Kusch C, Eiring P, Bryson S, Sauer M, Nieswandt 19 B et al (2019) Platelet lamellipodium formation is not required for thrombus formation and 20 stability. Blood 134: 2318-2329

21 Sinitski D, Kontos C, Krammer C, Asare Y, Kapurniotu A, Bernhagen J (2019) Macrophage Migration Inhibitory Factor (MIF)-Based Therapeutic Concepts in Atherosclerosis and Inflammation. Thromb Haemost 119: 553-566

24 Steen A, Larsen O, Thiele S, Rosenkilde MM (2014) Biased and g protein-independent 25 signaling of chemokine receptors. Front Immunol 5: 277 
1 Strüßmann T, Tillmann S, Wirtz T, Bucala R, von Hundelshausen P, Bernhagen J (2013)

2 Platelets are a previously unrecognised source of MIF. Thromb Haemost 110: 1004-1013

3 Struyf S, Salogni L, Burdick MD, Vandercappellen J, Gouwy M, Noppen S, Proost P,

4 Opdenakker G, Parmentier M, Gerard C et al (2011) Angiostatic and chemotactic activities of

5 the CXC chemokine CXCL4L1 (platelet factor-4 variant) are mediated by CXCR3. Blood 117:

$6 \quad 480-488$

7 Sun HW, Bernhagen J, Bucala R, Lolis E (1996) Crystal structure at 2.6-A resolution of 8 human macrophage migration inhibitory factor. Proc Natl Acad Sci U S A 93: 5191-5196

9 Tillmann S, Bernhagen J, Noels H (2013) Arrest functions of the MIF ligand/receptor axes in 10 atherogenesis. Front Immunol 4: 115

11 Tilstam PV, Qi D, Leng L, Young L, Bucala R (2017) MIF family cytokines in cardiovascular diseases and prospects for precision-based therapeutics. Exp Opin Ther Targ 21: 671-683

13 Vajda S, Yueh C, Beglov D, Bohnuud T, Mottarella SE, Xia B, Hall DR, Kozakov D (2017)

14 New additions to the ClusPro server motivated by CAPRI. Proteins 85: 435-444

15 von Hundelshausen P, Agten SM, Eckardt V, Blanchet X, Schmitt MM, Ippel H, Neideck C, 16 Bidzhekov K, Leberzammer J, Wichapong K et al (2017) Chemokine interactome mapping 17 enables tailored intervention in acute and chronic inflammation. Sci Trans/ Med 9

18 von Hundelshausen P, Petersen F, Brandt E (2007) Platelet-derived chemokines in vascular 19 biology. Thromb Haemost 97: 704-713 von Hundelshausen P, Weber C (2007) Platelets as immune cells: bridging inflammation and cardiovascular disease. Circ Res 100: 27-40

Wakasugi K, Schimmel P (1999) Two distinct cytokines released from a human aminoacyltRNA synthetase. Science 284: 147-151 
1 Weber C, Kraemer S, Drechsler M, Lue H, Koenen RR, Kapurniotu A, Zernecke A, 2 Bernhagen $J$ (2008) Structural determinants of MIF functions in CXCR2-mediated 3 inflammatory and atherogenic leukocyte recruitment. Proc Natl Acad Sci U S A 105: 16278$4 \quad 16283$

5 Weber C, Noels H (2011) Atherosclerosis: current pathogenesis and therapeutic options. Nat 6 Med 17: 1410-1422

7 Wirtz TH, Tillmann S, Strussmann T, Kraemer S, Heemskerk JW, Grottke O, Gawaz M, von 8 Hundelshausen P, Bernhagen J (2015) Platelet-derived MIF: a novel platelet chemokine with 9 distinct recruitment properties. Atherosclerosis 239: 1-10

Yan LM, Tatarek-Nossol M, Velkova A, Kazantzis A, Kapurniotu A (2006) Design of a mimic of nonamyloidogenic and bioactive human islet amyloid polypeptide (IAPP) as nanomolar affinity inhibitor of IAPP cytotoxic fibrillogenesis. Proc Natl Acad Sci U S A 103: 2046-2051

Zernecke A, Bernhagen J, Weber C (2008) Macrophage migration inhibitory factor in cardiovascular disease. Circulation 117: 1594-1602

Zhou M, Li Q, Wang R (2016) Current Experimental Methods for Characterizing ProteinProtein Interactions. ChemMedChem 11: 738-756

Ziegler-Heitbrock HW, Thiel E, Futterer A, Herzog V, Wirtz A, Riethmuller G (1988) Establishment of a human cell line (Mono Mac 6) with characteristics of mature monocytes. Int J Cancer 41: 456-461

Zlotnik A, Burkhardt AM, Homey B (2011) Homeostatic chemokine receptors and organspecific metastasis. Nat Rev Immunol 11: 597-606 


\section{Figures}

\section{Figure 1}

Unbiased chemokine protein array identifies CXCL4L1, but not CXCL4, as a novel interaction candidate of MIF. (A) Schematic illustrating binding of biotinylated MIF to the chemokine protein array. (B) Layout of the immobilized chemokines, atypical chemokines and alarmins (top) and membrane of chemokine solid phase assay performed at pH 8.0, developed against bound biotin-MIF (bottom). (C) Close-up of the membrane with a focus on CXCL4 and CXCL4L1 with the corresponding negative control membrane, incubated without biotin-MIF.

\section{Figure 2}

Validation of MIF/CXCL4L1 complex formation by a variety of protein-protein interaction assays and verification of the specificity of MIF complexation with CXCL4L1 over CXCL4. (A) Semi-endogenous pulldown assay, in which endogenous CXCL4L1 from MonoMac6 lysates was captured with recombinant biotinylated MIF and pulled down by streptavidin-coated paramagnetic beads. Blots, developed against MIF (left) and CXCL4L1 (right), show representative results of three independent experiments. Input corresponds to $5 \%$ cell lysate without pull-down and control (Ctrl) refers to pull-downs performed in the absence of biotin-MIF. Molecular weight markers were lelectrophoresed in the same gel and relevant marker sizes are indicated. (B) Interrogation of MIF/CXCL4L1 complex formation by surface plasmon resonance (SPR) spectroscopy using chip-immobilized biotin-MIF titred against increasing concentrations of CXCL4L1. Measurements indicate an interaction between MIF and CXCL4L1 with an estimated KD of \pm $n M$. The SPR response signal is given in relative units (RU). (C) Same as (B), except that titration was performed with CXCL4. Corresponding SPR spectroscopy data for MIF and CXCL4. No detectable binding signal was obtained and no KD could be derived. (D) Interrogation of MIF/CXCL4L1 complex formation by microscale thermophoresis (MST) utilizing fluorescently labeled MIF and CXCL4L1 in solution. MST analysis revealed a KD of $159.8 \pm 16.8 \mathrm{nM}$ for the interaction of MIF and CXCL4L1. (E) Same as (D), except that CXCL4 was tested. The derived apparent KD of $2.0 \pm 0.8 \mu \mathrm{M}$ was ten-fold higher compared to MIF/CXCL4L1.

\section{Figure 3}

Co-incubation with CXCL4L1 inhibits MIF-mediated immune cell chemotaxis. (A) Migration of human CD4+ T-cells embedded in a gel matrix, subjected to gradients of MIF, CXCL4L1 or both. Movement of cells was followed by live cell imaging and individual tracks reconstructed from acquired images. Tracks 
of cells migrating towards the indicated stimuli are marked in the corresponding color. Starting point was centered to $x=y=0$. The black crosshair indicates the cell population's center of mass after migration.

(B) Quantification of the 3D chemotaxis experiment in (A), indicating that complexation of MIF by CXCL4L1 attenuates MIF-mediated directed migration of human CD4+ T-cells. Plotted is the calculated forward migration index (FMI), based on manual tracking of at least individual cells per treatment. (C) Migration trajectories of murine microglia, obtained by live cell imaging for $15 \mathrm{~h}$, treated with MIF, CXCL4L1, or both. Used concentrations: MIF: nM, CXCL4L1: $1.6 \mathrm{nM} ; \mathrm{n}=5$ independent experiments; horizontal bar: $\mu \mathrm{m}$. (D) Analysis of microglia motility, based on each tracked cell accumulated distance, shown in (C). Data is presented as mean \pm SD. Statistical significance is indicated as described: ${ }^{*} P<$ $0.05 ; * \star, P<0.01 ; * \star \star, P<0.001$.

\section{Figure 4}

MIF/CXCL4L1 complex formation inhibits binding of MIF to CXCR4 and signaling of MIF through the CXCR4 signaling axis. (A) Fluorescence polarization (FP) spectroscopy shows the interaction of Alexa488-labeled MIF with the soluble CXCR4 receptor mimic msR4M-L1 with an apparent KD of $237.2 \pm$ $24.2 \mathrm{nM}$. Data is presented as mean of 23 independent experiments; error bars represent the SD. (B) Preincubation of MIF with CXCL4L1 (160-fold molar excess) prevents the interaction of MIF with msR4M-L1 (app. KD > $10 \mu \mathrm{M}$ ). Mean of 3 experiments \pm SD. (C) Dynamic mass redistribution (DMR) measurements with HEK293 cells stably expressing CXCR4 indicate that the cellular response to MIF is reduced, when MIF is pre-incubated with CXCL4L1. The DMR response of CXCR4-expressing HEK293 cells to MIF in the presence or absence of the CXCR4-1 antagonist AMD3100 is also shown, confirming the CXCR4dependency of the cellular response to MIF.

\section{Figure 5}

Co-localization and interaction of MIF and CXCL4L1 in human platelet preparations, detected in multiphoton microscopy (MPM). (A): MPM images of isolated platelets, forming small aggregates, stained for MIF and CXCL4L1. White arrowheads indicate areas of colocalization. Size bar: $5 \mu \mathrm{m}$. (B) MPM images of isolated, more separated platelets, stained as in (A), showing colocalization of MIF and CXCL4L1. Size bar: $\mu \mathrm{m}$. (C) Fluorescence lifetime imaging (FLIM) of platelets isolation as shown in (B). Color-code corresponds to lifetime of the donor, Alexa 488, the dye used for the antibody-based staining of MIF. (D) Histogram of the Förster Resonance Energy Transfer (FRET) efficiency in (C). (E) Donor lifetime shortening, presented as the mean lifetime $(\tau)$, average weighted, of the donor (Alexa 488, MIF staining) alone, and in combination with the acceptor fluorophore (Cy3, CXCL4L1 staining), where FRET occured. 


\section{Figure 6}

Proximity ligation assay (PLA) indicates that MIF/CXCL4L1 heterocomplexes are present in human thrombus tissue. (A) MIF/CXCL4L1 complex formation in thrombus specimen revealed by PLA. PLApositive signals are depicted in yellow; tissue was counterstained with fluorescent-labeled phalloidin (cyan). Stained tissue samples were imaged by CLSM; size bar: $50 \mu \mathrm{m}$. (B) HE staining of thrombus tissue specimen; size bar: $21 \mu \mathrm{m}$.

\section{Figure 7}

(A) Thrombus formation in human blood under flow stress is enhanced by MIF, and this effect is diminished by pre-incubation of MIF with CXCL4L1. Fluorescent staining with DiOC6. Shown are representative images of one experiment, performed at a shear rate of $s-1$; size bar: $100 \mu \mathrm{m}$. (B) Quantification of thrombi sizes from flow chamber experiments, as depicted exemplarily in (A). MIFmediated increase in thrombus-covered area is diminished, when MIF is pre-incubated with CXCL4L1. $\mathrm{n}=$ 6 experiments and platelets coming from donors. (C) Quantification of total thrombi numbers per treatment group. As thrombus numbers remain unchanged, effects on thrombus-covered area originate from the size of the formed thrombi (see also Supplementary Figure 5); $n=$ experiments. (D) Analysis and quantification of platelet morphology upon adhesion on fibrinogen-coated coverslips. Activated platelets were allowed to adhere on fibrinogen-coated coverslips that were pre-treated with MIF, CXCL4L1 or a mixture of both for the indicated times. After fixing with PFA, images of randomly selected areas were taken and platelet morphology analyzed. Treatment with a combination of MIF and CXCL4L1 led to a reduction in the large lamellopodia phenotype, favoring small lamellopodia, with the MIF/CXCL4L1 complex showing a stronger effect then the individual proteins; $n=6$ experiments. (E) Platelet morphology distribution after min for each treatment group according to panel (D).

\section{Figure 8}

Summary scheme and suggested model of CXCL4L1/MIF complex formation and functions. The atypical chemokine MIF and the classical chemokine CXCL4L1, e.g. present in an inflammatory or atherogenic microenvironment after release from platelets, form heteromeric complexes. Complexes inhibit inflammatory effects of MIF on leukocyte recruitment as well as its pro-thrombotic effects through impairing MIF interactions with its non-cognate receptor CXCR4.

\section{Supplementary Files}

This is a list of supplementary files associated with this preprint. Click to download. 
- BrandhoferetalCMLSSupplFigures.pdf 